\title{
A tale of two tails and an off-centered envelope: diffuse light around the CD galaxy NGC 3311 in the Hydra I cluster ${ }^{\star}$
}

\author{
M. Arnaboldi ${ }^{1,2}$, G. Ventimiglia' ${ }^{1,3}$, E. Iodice ${ }^{4}$, O. Gerhard ${ }^{3}$, and L. Coccato ${ }^{1,3}$ \\ ${ }^{1}$ European Southern Observatory, Karl-Schwarzschild-Str. 2, 85748 Garching, Germany \\ e-mail: marnabol@eso.org \\ 2 INAF, Osservatorio Astronomico di Pino Torinese, 10025 Pino Torinese, Italy \\ 3 Max-Planck-Institut für Extraterrestrische Physik, Postfach 1312, Giessenbachstr., 85741 Garching, Germany \\ ${ }^{4}$ INAF, Osservatorio Astronomico di Capodimonte, 80126 Napoli, Italy
}

Received 19 February 2011 / Accepted 25 June 2012

\begin{abstract}
Context. The formation of intracluster light and extended halos around brightest cluster galaxies is closely related to morphological transformation, tidal stripping, and the disruption of galaxies in clusters.

Aims. Here we look for observational evidence to characterize these processes, by studying the morphology and kinematics of the diffuse light in the core of the Hydra I cluster.

Methods. With $V$-band surface photometry, we derive the structural parameters (Sersic index $n$, effective radius $R_{\mathrm{e}}, b / a$, and major axis position angle PA) of the two giant elliptical galaxies NGC 3311 and NGC 3309 in the cluster core. We construct a two-dimensional photometric model, and investigate the diffuse light structures in the residual image after subtracting the two-dimensional model. We also analyze deep long-slit spectra, and establish a link between the structures in the light distribution, the absorption-line kinematics, and the line-of-sight velocity distributions of nearby galaxies and planetary nebulae (PNs).

Results. The central galaxy NGC 3311 is surrounded by an extended, symmetric outer halo with $n=10$ and an additional, offcentered envelope whose centroid is shifted by about $50^{\prime \prime}$ to the north-east. Its luminosity $L_{\mathrm{V}}=1.2 \times 10^{10}\left( \pm 6.0 \times 10^{8}\right) L_{\odot}$ corresponds to $\sim 50 \%$ of the luminosity of the symmetric halo in the same region $(\sim 15 \%$ of its entire luminosity). The velocity dispersion of the halo rises to cluster core values, $\sim 400-500 \mathrm{~km} \mathrm{~s}^{-1}$, for $R>20^{\prime \prime}$. On the basis of measured PN velocities, at least part of the offcentered envelope consists of high-velocity accreted stars. We also discover two tidal streams in the cluster center, emerging from the dwarf galaxy HCC 026 and the S0 galaxy HCC 007. The HCC 026 stream is redshifted by $\sim 1200 \mathrm{~km} \mathrm{~s}^{-1}$ with respect to NGC 3311 $\left(V_{\mathrm{N} 3311} \simeq 3800 \mathrm{~km} \mathrm{~s}^{-1}\right)$, as for HCC 026 itself, a fraction of PNs in the off-centered envelope, and several other dwarf galaxies nearby. The stars in one of the HCC 026 tails are known to be consistent with the low-metallicity population of HCC 026, and our photometry shows that this galaxy is almost dissolved by the tidal field. The tidal stream around HCC 007 extends over at least $\sim 110 \mathrm{kpc}$, is fairly thick, and is brighter on the side of the asymmetric outer halo of NGC 3311, which it may join. Its luminosity is several $10^{9} L_{\odot}$, similar to the luminosity of the stripped-down galaxy HCC 007. The redshift of the stream is determined from a few PN velocities and is similar to those of both HCC 007 and HCC 026.

Conclusions. An entire group of small galaxies is currently falling through the core of the Hydra I cluster; these galaxies have already been partially dissolved by the strong tidal field. Their light is being added to the outer halo and intracluster light around the $\mathrm{cD}$ galaxy NGC 3311. The Hydra I cluster provides a vivid example of the morphological transformation and tidal dissolution of galaxies in clusters.
\end{abstract}

Key words. galaxies: clusters: general - galaxies: clusters: individual: Hydra I - galaxies: kinematics and dynamics galaxies: individual: NGC 3311

\section{Introduction}

Galaxy clusters are the most massive virialized structures in the universe, and may consist of thousands of galaxies. One of the most interesting fields in modern cosmology is the study of the mechanisms for the growth and evolution of such systems and the evolution of galaxies within them. The hierarchical model predicts that structure formation and evolution occurs through the merging of smaller units into larger systems, and this model has been supported by much observational evidence. The observational appearance of clusters and their galaxies depends additionally on the evolution of the baryonic component, which is less well understood. As galaxies fall into dense environments,

\footnotetext{
* Based on observations collected at the European Organization for Astronomical Research in the Southern Hemisphere, Chile, under the observing programs 082.A-0255(A), 076.B-0641(A), 065.N-0459.
}

their evolution is affected by a variety of dynamical processes such as tidal interaction and harassment, mergers and cannibalism, gas stripping and starvation (see Poggianti 2004; De Lucia 2007 , for a review). Which of these mechanisms takes the leading role for a given galaxy morphological type in different environmental conditions still remains to be understood.

In the nearby universe, the evolution of clusters as a whole and the evolution of galaxies in clusters can be addressed by studying the dynamics of the intracluster light (ICL). The ICL is the diffuse light in galaxy clusters emitted by stars that are not bound to any specific galaxies; for a review of the subject we refer to Arnaboldi \& Gerhard (2010). Wide-field surface photometry shows structures in the ICL on all scales, from a few arcminutes to degrees on the sky (Thuan \& Kormendy 1977; Mihos et al. 2005; Rudick et al. 2009). Recent studies have shown that the ICL provides direct evidence for the dynamical status of 
galaxy cluster cores (Gerhard et al. 2007; Doherty et al. 2009; Ventimiglia et al. 2011), because it contains the fossil record of past interactions, owing to its long dynamical time.

Cosmological hydro-dynamical simulations predict that the ICL is formed by stars that are unbound from galaxies during the interactions they experience as they fall through the cluster potential well and interact with other cluster galaxies and the cluster tidal field. In these simulations, the ICL shows significant substructures on all scales in its spatial and velocity distribution (Napolitano et al. 2003; Murante et al. 2004; Willman et al. 2004; Sommer-Larsen et al. 2005). During the intial phases of its formation, the ICL morphology is dominated by long, linear features such as streams that become more diffuse at later times as they spread in the cluster volume (Rudick et al. 2009). Most of the simulated intracluster stars become separated from their parent galaxies during the merging history leading to the formation of the brightest cluster galaxy (BCG) at the cluster center, while for the production of the ICL at larger radii other mechanisms such as tidal stripping are more important (Murante et al. 2007; Puchwein et al. 2010).

In this paper, we report on surface photometry and longslit spectroscopy of the ICL at the center of the Hydra I cluster, a medium, compact cluster at a $\sim 50 \mathrm{Mpc}$ distance in the southern hemisphere, that has a central cD galaxy, NGC 3311. The aim is to compare the structures in the surface brightness distribution in the cluster core around NGC 3311 with kinematic information, including also the line-of-sight (LOS) velocities of intracluster planetary nebulas (ICPNs). Studying the kinematics of the ICL in nearby clusters such as Hydra I is possible with ICPNs because (i) these objects are relatively easy to detect owing to their strong [OIII] emission line (Jacoby 1989; Ciardullo et al. 1989); and (ii) they are good tracers of the light distribution of the parent stellar population (Buzzoni et al. 2006; Coccato et al. 2009). The LOS velocity distribution (LOSVD) of the ICPNs associated with the diffuse light in the central $100 \mathrm{kpc}$ around NGC 3311 was determined by Ventimiglia et al. (2008, 2011). They detected discrete velocity components at redshifts $\sim 1800 \mathrm{~km} \mathrm{~s}^{-1}$ and $\sim 5000 \mathrm{~km} \mathrm{~s}^{-1}$, in addition to a broad component with a velocity dispersion $\sigma \simeq 500 \mathrm{~km} \mathrm{~s}^{-1}$ at approximately the systemic velocity of the Hydra I cluster $\left(\simeq 3900 \mathrm{~km} \mathrm{~s}^{-1}\right)$. Ventimiglia et al. (2011) concluded that the broad velocity component in the ICPN LOSVD may trace the high-velocity-dispersion outer halo of NGC 3311 (Ventimiglia et al. 2010), while the discrete components trace sub-components that fell through the cluster core, were tidally disrupted, and have not yet dynamically mixed in the gravitational potential.

This paper is structured as follows: in Sect. 2, we present optical $V$-band images for the Hydra I cluster core. Isophote fitting and analysis of the surface brightness profiles of the galaxies NGC 3311 and NGC 3309 is carried out in Sect. 3. Two-dimensional models for the optical data are derived in Sect. 4, showing the existence of an off-centered outer halo around NGC 3311 as well as tidal streams superposed on this halo. In Sect. 5, long-slit spectroscopic data and kinematic measurements for the halo of NGC 3311 and the localized stream about HCC 026 are presented and discussed. In Sect. 6, we investigate the correspondence between the photometric components and kinematic substructures in the velocity distribution of Hydra I PNs, and both dwarf and S0 galaxies within $50 \mathrm{kpc}$ of the center of NGC 3311. Section 7 briefly discusses the peculiar outer halo of NGC 3311, the properties of the newly discovered tidal streams, and the formation of ICL from this group of galaxies in disruption. Finally, Sect. 8 contains our summary and conclusions. We assume a distance to the Hydra I cluster of $D=50 \mathrm{Mpc}$, so $1^{\prime \prime}=0.247 \mathrm{kpc}$.

\section{Optical imaging of the Hydra I cluster core}

The core of the Hydra I cluster is dominated by two giant elliptical galaxies, NGC 3311 and NGC 3309. Early CCD surface photometry showed that both NGC 3311 and NGC 3309 are fitted by an $R^{1 / 4}$ law within a $30^{\prime \prime}$ distance from their centers (Vasterberg et al. 1991). On the basis of the large $R_{\mathrm{e}}=98^{\prime \prime}$ value derived from their $R^{1 / 4}$ fit, Vasterberg et al. (1991) classified NGC 3311 as a cD galaxy. At the center of this galaxy, a complex dust lane is found, and the observed surface brightness profile is fainter than the best-fit $R^{1 / 4}$ profile. Both the dust lane and the core in the central profile are confirmed by HST WFPC2 imaging (Laine et al. 2003). NGC 3309 does not have a dust lane at its core (Vasterberg et al. 1991). Misgeld et al. (2008) published VLT/FORS1 images of the Hydra cluster in the $V$-band, that were used by Richtler et al. (2011) to derive the average, radial surface brightness profile for NGC 3311 down to $\mu_{V}=$ $26.5 \mathrm{mag} \mathrm{arcsec}^{-2}$. They classified NGC 3311 as a cD galaxy because of its low central surface brightness and extended radial profile.

In the present work, we derive accurate quantitative photometry for the galaxies NGC 3311 and NGC 3309 and the diffuse light at the Hydra I core. In what follows, we use two data sets for this purpose: VLT/FORS1 archival data for the center of the Hydra I cluster and $V$ band images obtained with WFI at the ESO/MPI $2.2 \mathrm{~m}$ telescope. These $V$ band images allow us to measure the profile shape at large radii where the surface brightness is low, and analyze the substructures in the light distribution. The VLT/FORS1 and $2.2 \mathrm{~m} / \mathrm{WFI}$ data contain complementary information: the FORS 1 data are deep, and allow reliable measurements down to $\mu_{V}=26.5 \mathrm{mag} \operatorname{arcsec}^{-2}$ (Richtler et al. 2011), while the WFI data with their large field-of-view provide a robust estimate of the sky background.

\subsection{VLT/FORS1 deep $V$ band photometry - observations and data reduction}

We retrieved Johnson $V$ band imaging data for NGC 3311 and NGC 3309 from the ESO Science Archive Facility, that had been acquired with VLT/FORS1 in service mode on April 04, 2000 (program 65.N-0459(A), PI Hilker). The VLT/FORS1 images cover a field-of-view (FoV) of $6.8^{\prime} \times 6.8^{\prime}$ at an angular scale of 0.2 pixel $^{-1}$. During the observations, the FoV was centered at $\alpha=10^{\mathrm{h}} 36^{\mathrm{m}} 36.14^{\mathrm{s}}, \delta=-27^{\mathrm{d}} 32^{\mathrm{m}} 51^{\mathrm{s}}$, avoiding a bright star NE of NGC 3311. The dataset included three exposures of $480 \mathrm{~s}$ each for a total observing time of $0.4 \mathrm{~h}$. The resulting average seeing in the combined median image is $F W H M \sim 0 . ' 6$, and both the central $\sim 5^{\prime \prime}$ diameter region of NGC 3311 and the $\sim 8^{\prime \prime}$ diameter region of NGC 3309 are saturated.

Standard calibration, bias, and sky flat were also retrieved from the ESO Archive. Several Landolt standard stars in the Rubin 152 field had been observed in $V$ band to perform the photometric calibration. The zero point for the $V$ band VLT/FORS1 photometry $Z P_{V, \mathrm{FORS} 1}=27.43 \pm 0.06$ was derived independently for the present work. The correction for extinction in the $V$ band amounts to 0.25 mag (Schlegel et al. 1998).

The instrumental signatures in the VLT/FORS1 images were removed using standard Image Reduction and Analysis Facility (IRAF) tasks. Spikes from saturated stars in the field were also removed (see Sect. 2.2). Images were registered before 

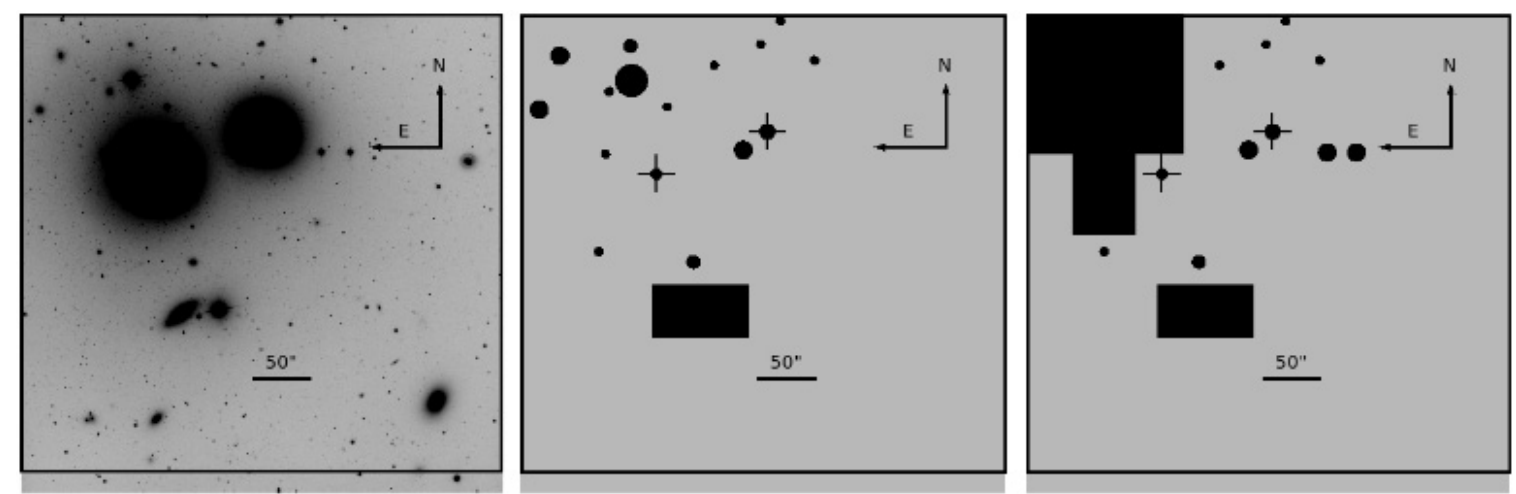

Fig. 1. Left panel - VLT/FORS1 $V$ band co-added image of the Hydra I cluster core with NGC 3311 and NGC 3309 visible in the upper part of the image. A two dimensional (2D) photometric model is fit within the region of $6.8 \times 6.4$ limited by the box (see Sect. 4.1 for details). Middle panel - The mask "cmask" adopted for the 2D-model fit of the VLT/FORS1 data, which masks stars and galaxies in the chosen region as well as the centers of NGC 3311 and NGC 3309. Right panel - The mask "allmask" for the 2D fit that also masks part of the north-east regions, in addition to the stars and galaxies in "cmask" (see Sect. 4.1 for details). In the middle and right panels, the two crosses indicate the positions of the centers of the two giant galaxies.

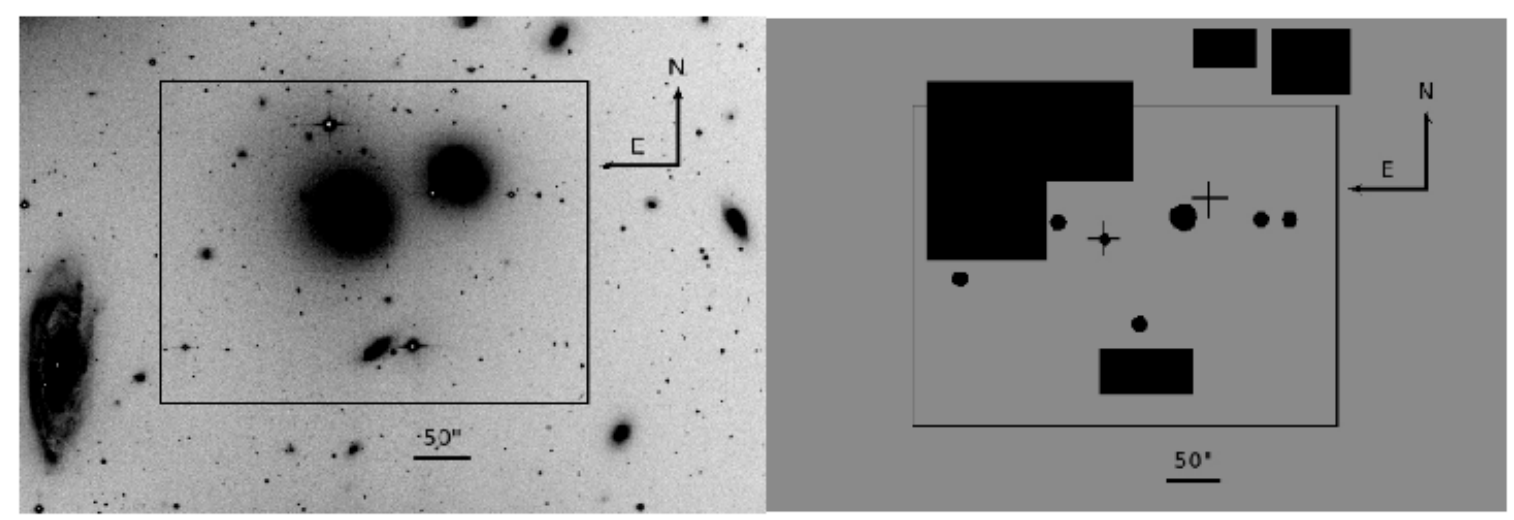

Fig. 2. Left panel - $2.2 \mathrm{~m} /$ WFI $V$ band image of the Hydra I cluster core; NGC 3311 is visible on the left and NGC 3309 on the right. A 2D-photometric model is fit within the region of 6'.4 $\times 4.8$ limited by the box. Right panel - The mask "allmask" adopted for the fit to the bright central regions (see Sect. 4.2 for details). The two crosses in the right panel indicate the positions of the centers of NGC 3311 and NGC 3309.

co-addition; the co-added final VLT/FORS1 $V$ band image is shown in Fig. 1.

\subsection{Wide Field Imager $V$ band photometry - observations and data reduction}

Johnson $V$ band imaging of the Hydra I cluster was acquired in service mode on the night of January 12, 2006 at the Wide Field Imager (WFI) on the ESO/MPI $2.2 \mathrm{~m}$ telescope, at the La Silla observatory. The WFI imager covers a field-of-view (FoV) of $34^{\prime} \times 33^{\prime}$ with a mosaic of $4 \times 2$ CCDs $(2 \mathrm{k} \times 4 \mathrm{k})$ at an angular scale of 0.238 pixel $^{-1}$. During the observations, the FoV was centered at $\alpha=10^{\mathrm{h}} 36^{\mathrm{m}} 51^{\mathrm{s}}, \delta=-27^{\mathrm{d}} 31^{\mathrm{m}} 35^{\mathrm{s}}$. Thirteen exposures of $300 \mathrm{~s}$ each were obtained for a total observing time of $\sim 1.1 \mathrm{~h}$. The resulting average seeing in the combined median image is $F W H M \sim 0.7$.

Standard calibration, bias, sky flat and dark sky exposures were also obtained. Several Landolt standard stars in the Rubin 149 field were observed in $V$ band for the photometric calibration. The zero point for the $V$ band photometry is $Z P_{V}=$ $24.02 \pm 0.02$.

Data reduction was carried out with standard IRAF tasks for pre-reduction and calibration. After bias subtraction and flat fielding, the frames were corrected for any remaining residuals by using dark sky exposures. The average background emission was then measured in several regions of the FoV far from the galaxy light and the final average value was subtracted from each single frame. The IRAF task NOAO.NPROTO.IRMOSAIC was used to obtain a mosaic of the CCDs. Each frame was inspected and bright spikes associated with saturated stars were removed by linear interpolation from nearby columns/rows before the frames were co-added. Residual sky offsets with respect to the average value were removed before the CCD frames were mosaiced. Finally, the mosaic images were registered. Image fluxes were then scaled to a reference image to account for transparency variations, following which images were co-added with a threshold rejection implemented to reduce the effects of the CCD gaps. The final co-added image is shown in the left panel of Fig. 2.

As a first step in the study of the light distribution in NGC 3311, we determine the extension of the dust lane in its central region. We use the FMEDIAN task in IRAF with a smoothing box of $15 \times 15$ pixels, and compute the ratio of the $V$ band image to its FMEDIAN smoothed version; the resulting unsharp-masked $V$-band image is shown in Fig. 3.

This figure illustrates the presence of several distinct components at the center of NGC 3311. A complex dust lane crosses the galaxy center in the direction north-south (NS) in the high angular-resolution image in Laine et al. (2003). Bright knots are 


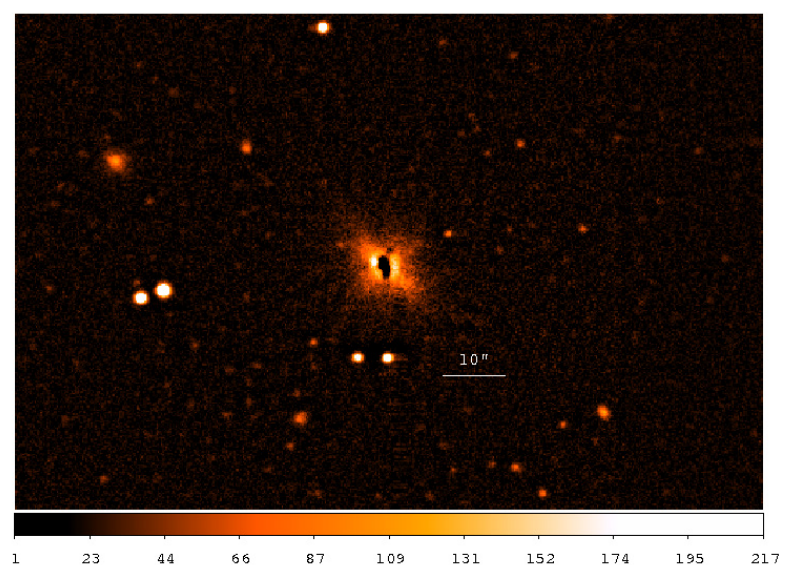

Fig. 3. Unsharp-masked $V$ band image obtained from optical data acquired at the ESO/MPI $2.2 \mathrm{~m}$ telescope with the WFI; North is up, and east to the left. The white bar indicates a $10^{\prime \prime}$ length. The inner dust lane at the center of NGC 3311 is about $2^{\prime \prime}$ wide and 7" long (Laine et al. 2003), and is embedded within a central light excess of about $16^{\prime \prime}$ in diameter, where bright knots are visible here and in the HST image of Laine et al. (2003).

seen east and southwest of the galaxy center, within and around the dust lane out to $8^{\prime \prime}$ in radius. According to Vasterberg et al. (1991), the dominant knot is bluer, $\Delta(B-r)=-0.10$, than the surrounding stellar population.

\section{Surface photometry of NGC 3311 in the $V$ band}

Here we describe our isophotal analysis of the light distribution of NGC 3311 from the $V$-band data. We then derive the $V$-band surface brightness profiles along several specific directions through the galaxy to characterize the deviations from a simple, concentric, elliptical-light distribution.

\subsection{Isophote fitting}

We applied the ELLIPSE task in IRAF to the $V$ images to perform the isophotal analysis of NGC 3311. The position angle (PA), ellipticity, and average surface brightness profiles of NGC 3311 are shown in Fig. 4.

The ELLIPSE $V$-band average surface brightness profile extends out to $80^{\prime \prime}$ from the galaxy center before the halo light from NGC 3309 becomes significant. We note the excellent agreement between the VLT/FORS1 and WFI surface brightness profiles. The total integrated magnitudes within circular apertures, whose radius corresponds to the last measured point, are $m_{V}^{\mathrm{WFI}}\left(R=77^{\prime \prime} .4\right)=11.55 \mathrm{mag}$ and $m_{V}^{\mathrm{FORS}}\left(R=71^{\prime \prime} .5\right)=$ $11.36 \mathrm{mag}$. The half-light radii evaluated from the light-growth curves out to the last measured point are $R_{g, V}^{\mathrm{WFI}}=36^{\prime \prime} \pm 2^{\prime \prime}$ $(\sim 8.9 \mathrm{kpc})$ and $R_{g, V}^{\mathrm{FORS}}=32^{\prime \prime} \pm 2^{\prime \prime}(\sim 7.9 \mathrm{kpc})$.

At $R \leq 5^{\prime \prime}$, the presence of a dust-lane is indicated by the large variations in the $V$-band ellipticity and PA profiles. Between $5^{\prime \prime}$ and $15^{\prime \prime}$, the $V$-band isophotes twist by about 20 degrees owing to the inner bright knots seen around the dust lane (see Sect. 2.2). A twist of the NGC 3311 isophotes was also reported by Vasterberg et al. (1991), but the PA variation was not quantified. For radii $15^{\prime \prime}<R<45^{\prime \prime}$, the $V$-band ellipticity and PA profiles are nearly constant, with average values $\epsilon \simeq 0.05$ and $\mathrm{PA} \simeq 32^{\circ}$, i.e., the isophotes in this region are nearly round.

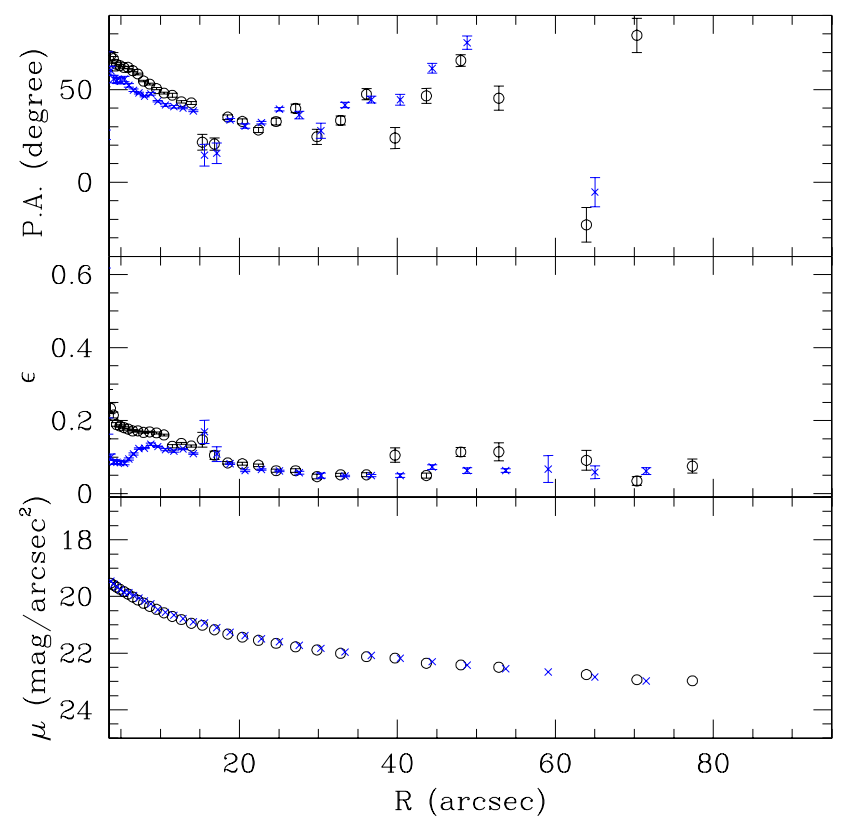

Fig. 4. One dimensional profiles of the PA of the isophotal major axis, ellipticity, and surface brightness obtained with ELLIPSE from $V$-band images of NGC 3311 (VLT/FORS1 blue crosses, $2.2 \mathrm{~m} /$ WFI black open dots). Because of the central dust lane, the profiles are well defined only outside 3 ". 5 .

\subsection{Analysis of surface brightness profiles along the principal axes of the light distribution}

The position angle profile from the isophote fit in Fig. 4 has a complicated radial dependence, which we now show is related to asymmetries in the light distribution. To this end, we investigate separately the surface brightness profiles along several axes across the light distribution in NGC 3311. From the isophotal fitting, the major axis is at $\mathrm{PA}=32^{\circ}$ and the minor axis at $\mathrm{PA}=122^{\circ}$. Along these directions, we extracted onedimensional (1D) surface brightness profiles from the $V$-band VLT/FORS1 and $2.2 \mathrm{~m} / \mathrm{WFI}$ images.

The $V$ band surface brightness profiles are asymmetric at $R>$ $30^{\prime \prime}$, as shown in Figs. 5 and 6. The major-axis profile at PA = $32^{\circ}$ (north-east - NE) is up to one magnitude brighter than that at $\mathrm{PA}=212^{\circ}$ (south-west $-\mathrm{SW}$ ) in the radial range $20^{\prime \prime}<R<$ $120^{\prime \prime}$ (Fig. 5). There is additional light along PA $=212^{\circ}$ at $R \geq$ $100^{\prime \prime}$, that may be related to a substructure in the Hydra I core. We investigated this idea by examining the residual image, once the light distributions of the bright galaxies had been subtracted. The light profiles along the minor axis, at $\mathrm{PA}=122^{\circ}$ (southeast $-\mathrm{SE}$ ) and $\mathrm{PA}=302^{\circ}$ (north-west $-\mathrm{NW}$ ), are symmetric for $R<60^{\prime \prime}$ (Fig. 6). At PA $=302^{\circ}$ and $R>60^{\prime \prime}$, we see the light from the NGC 3309 halo. For $R \leq 5^{\prime \prime}$, both $V$ band profiles showed the absorption by the central dust-lane.

In addition, we extracted folded profiles along the NS (PA = $\left.0^{\circ}\right)$ and $\mathrm{EW}\left(\mathrm{PA}=90^{\circ}\right)$ directions. These are also asymmetric (see Figs. 7 and 8). At $\mathrm{PA}=0^{\circ}$ and $R \geq 20^{\prime \prime}$, the galaxy becomes brighter than at $\mathrm{PA}=180^{\circ}$; the difference is about $0.5 \mathrm{mag}$ at $R=80^{\prime \prime}$ (Fig. 7). At $\mathrm{PA}=90^{\circ}, 270^{\circ}$ the profiles are symmetric for $R \leq 30^{\prime \prime}$, while in the radial range $30^{\prime \prime} \leq R \leq 60^{\prime \prime}$ the profile at $\mathrm{PA}=90^{\circ}$ is brighter (by about $0.2 \mathrm{mag}$ at $R \sim 50^{\prime \prime}$ ) than that at $\mathrm{PA}=270^{\circ}$. At larger radii the profile is affected by the light of NGC 3309. Figures 7 and 8 illustrate that the additional light is distributed over an opening angle larger than $90^{\circ}$ as seen from 
M. Arnaboldi et al.: Two tails and an off-centered envelope in the Hydra I cluster core

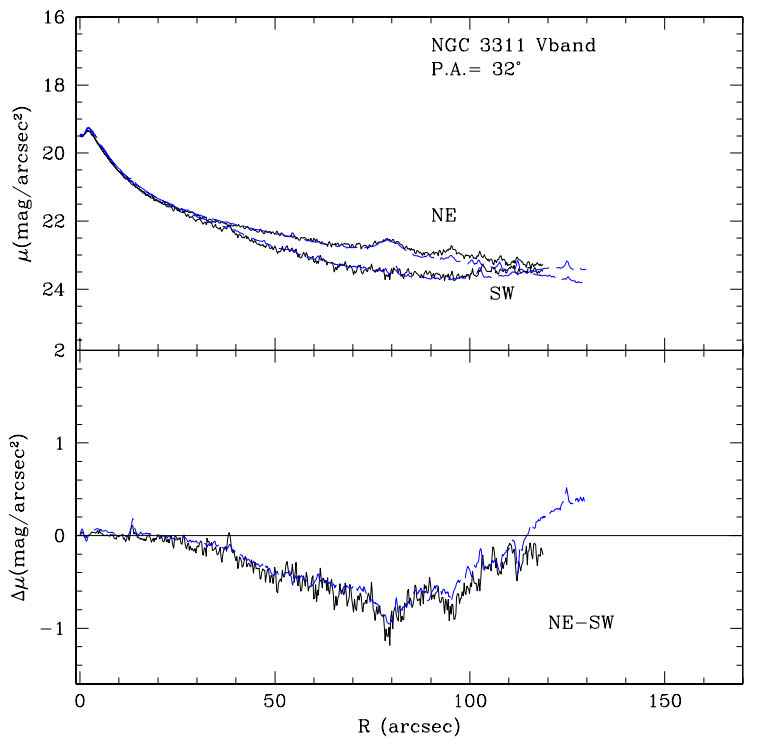

Fig. 5. Upper panel: folded $V$-band profiles extracted along $\mathrm{PA}=32^{\circ}$ (major axis, $2.2 \mathrm{~m} /$ WFI black full lines, VLT/FORS1 blue dashed lines). North-east is along $\mathrm{PA}=32^{\circ}$ and $\mathrm{SW}$ along $\mathrm{PA}=212^{\circ}$. Lower panel : difference profiles. The folded profile along the major axis illustrates the excess of light in the NE quadrant of the NGC 3311 halo, in the range of radii $20^{\prime \prime}<R<120^{\prime \prime}$, with a maximum excess of about one magnitude at $R=80^{\prime \prime}$.

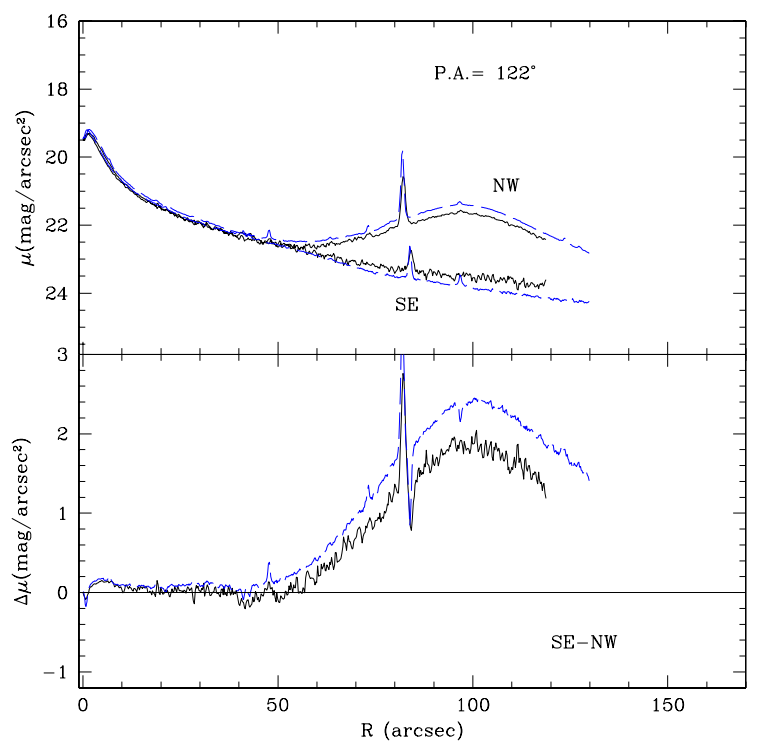

Fig. 6. Same as Fig. 5. Upper panel: folded $V$-band profiles extracted along PA $=122^{\circ}$ (minor axis, $2.2 \mathrm{~m} /$ WFI black full lines, VLT/FORS1 blue dashed lines). South-east is along PA $=122^{\circ}, \mathrm{NW}$ along PA $=$ $302^{\circ}$. Lower panel: difference profiles. On the NW side, for $R \geq 65^{\prime \prime}$ we see the contribution of the outer regions of NGC 3309.

the center of NGC 3311, therefore the substructure leading to the asymmetry in Fig. 5 is neither a radial stream nor fan.

The VLT/FORS1 and $2.2 \mathrm{~m} / \mathrm{WFI}$ profiles in Figs. 5 and 7 show very good agreement on both the N/NE and S/SW sides of NGC 3311 in addition to the difference profiles. This shows that systematic effects caused by scattered light from the bright star NE of NGC 3311 are negligible and that there are no residual gradients owing to the sky background along these directions. Figures 6 and 8 show a small relative variation between

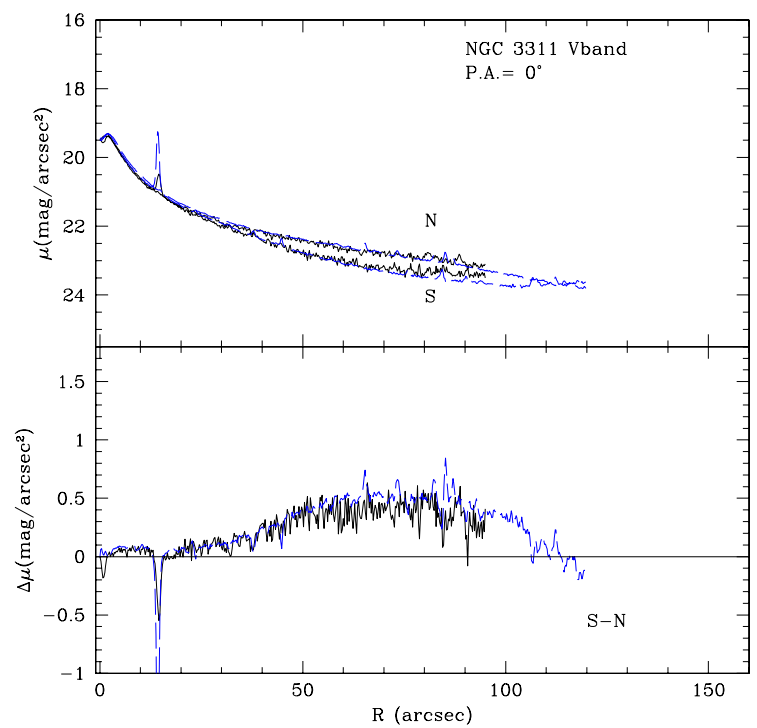

Fig. 7. Same as Fig. 5. Upper panel: folded $V$-band profiles extracted along PA $=0^{\circ}(2.2 \mathrm{~m} /$ WFI black full lines, VLT/FORS1 blue dashed lines). North is along $\mathrm{PA}=0^{\circ}, \mathrm{S}$ along $\mathrm{PA}=180^{\circ}$. Lower panel: difference profiles.

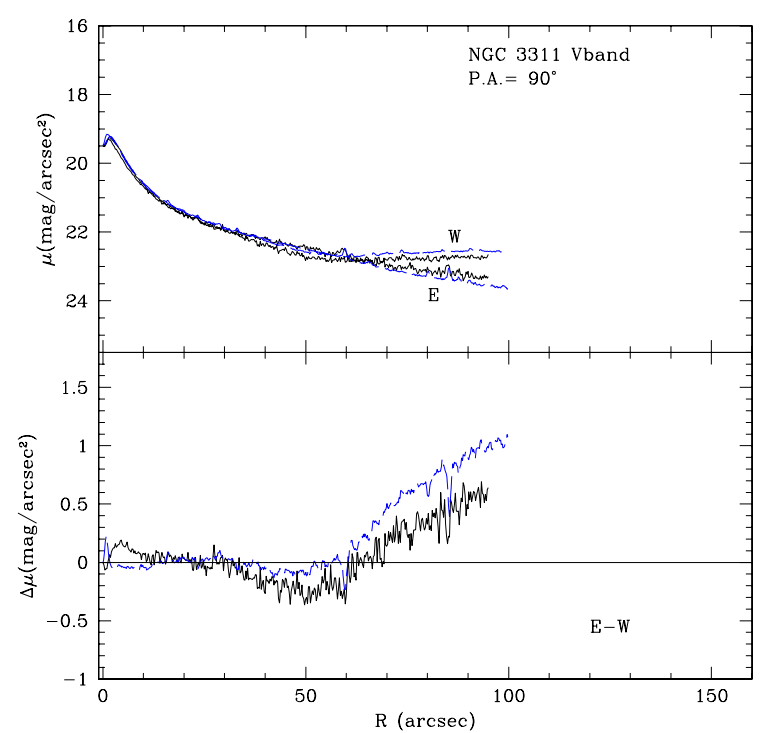

Fig. 8. Same as Fig. 5. Upper panel: folded $V$-band profiles extracted along $\mathrm{PA}=90^{\circ}(2.2 \mathrm{~m} /$ WFI black full lines, VLT/FORS1 blue dashed lines). East is along $\mathrm{PA}=90^{\circ}, \mathrm{W}$ along $\mathrm{PA}=270^{\circ}$. Lower panel: difference profiles.

the W/NW and E/SE sides, amounting to $~ 0.2$ mag over $200^{\prime \prime}$. As we discuss in Sect. 4.2, this does not affect our main results.

The analysis presented in this section illustrates that the halo of NGC 3311 is asymmetric. Between PA $=0^{\circ}$ and $\mathrm{PA}=90^{\circ}$, and in the radial range $30^{\prime \prime}<R<120^{\prime \prime}$, it is brighter than in the other three quadrants.

\section{Models of the light distribution in NGC 3311, NGC 3309, and the residual diffuse light in the Hydra I cluster core}

We constructed two-dimensional (2D) surface brightness models of the light distribution in the Hydra cluster core around NGC 3311. Any such model must include the light distribution of NGC 3309, because the outer regions of the two elliptical 

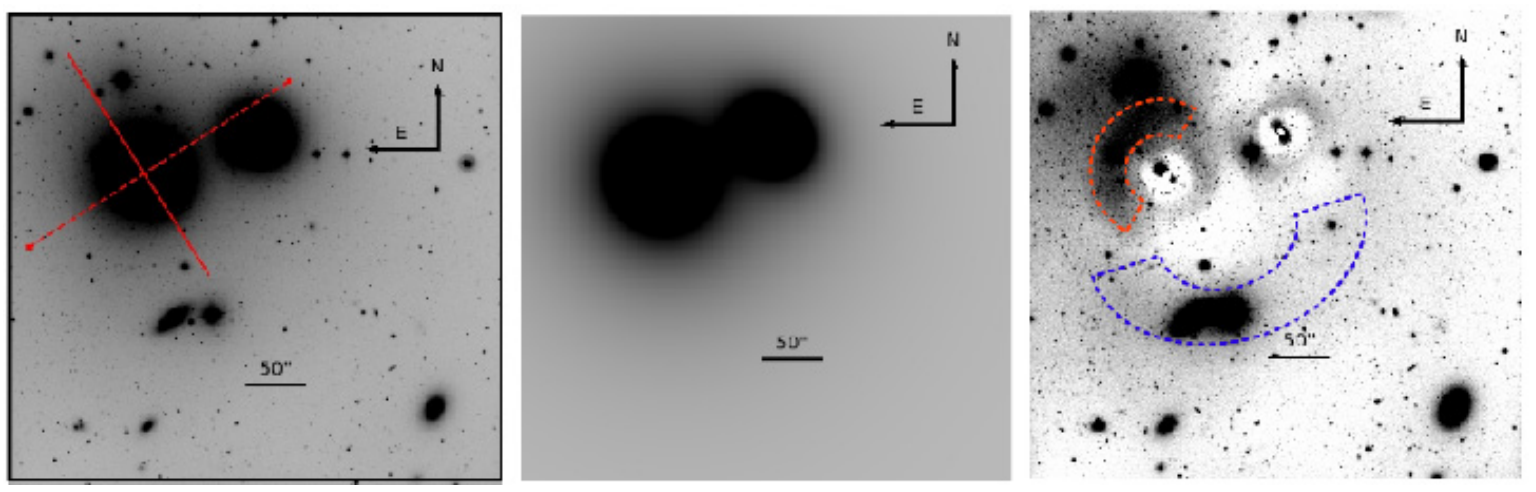

Fig. 9. GALFIT model of the VLT/FORS1 image obtained with "allmask" (see right panel of Fig. 1). Left panel: VLT/FORS1 $V$ band image. Full and dashed red lines indicate the major axis at $\mathrm{PA}=32^{\circ}$, and the minor axis, respectively. Center panel: combined 2D GALFIT models for NGC 3311 and NGC 3309. Right panel: residual image, showing extra light associated with the NE outer halo of NGC 3311, a region of negative residuals $\sim 60^{\prime \prime}$ south west of NGC 3311, and preliminary evidence of two tidal streams around HCC 026 and HCC 007 , outlined by the red- and blue dashed curves. The image size in all panels is $6.8 \times 6.4$, and darker colors indicate brighter regions.

Table 1. Parameters for the 2D GALFIT models of NGC 3309.

\begin{tabular}{lccc}
\hline \hline Parameter & $\begin{array}{c}V \text { band } \\
\text { FORS1 }\end{array}$ & $\begin{array}{c}V \text { band } \\
\text { FORS1 }\end{array}$ & $\begin{array}{c}V \text { band } \\
\text { FORS1 } \\
\text { max. symmetr. }\end{array}$ \\
\hline Comp. type & Sersic & Sersic & $\begin{array}{c}\text { Sersic } \\
m_{\text {tot }}\end{array}$ \\
$R_{\mathrm{e}}$ & $21.6 \pm 0.05$ & $11.6 \pm 0.05$ & $11.2 \pm 0.01$ \\
$n$ & $3.3 \pm 0.02$ & $21^{\prime \prime} .9 \pm 0.07$ & $35^{\prime \prime} \pm 0.25^{\prime \prime}$ \\
$b / a$ & $0.87 \pm 0.01$ & $0.85 \pm 0.02$ & $5.7 \pm 0.04$ \\
PA & $48.2 \pm 0.2$ & $50.7 \pm 0.2$ & $0.87 \pm 0.01$ \\
mask & "cmask" & "allmask" & "allmask" \\
\hline
\end{tabular}

Notes. Apparent $V$ band total magnitudes are in agreement with the published NED values (RC3).

galaxies overlap along the LOS. Furthermore, the modeling must also account for the asymmetric bright component in the $\mathrm{NE}$ outer region of NGC 3311, that is indicated by the asymmetry of the extracted profiles in Sect. 3.2.

\subsection{V-band 2D model for NGC 3311 and NGC 3309}

We modeled the surface brightness distributions of NGC 3311 and NGC 3309 from the $V$ band VLT/FORS1 image. As a model to fit to the VLT/FORS1 data, we first adopted the mask shown in the middle panel of Fig. 1, which is refereed to as "cmask" in what follows. It masks the saturated/bright stars in the field, the central $R<5^{\prime \prime}$ of NGC 3311, a region $R<8^{\prime \prime}$ around the saturated center of NGC 3309, and the background galaxies.

For the "cmask" model fit, the 2D Sersic model has parameters $n_{V}=3.3, R_{\mathrm{e}}=21^{\prime \prime}$. 1 for NGC 3309 and $n_{V}=5.0, R_{\mathrm{e}}=$ 270.' 6 for NGC 3311 (see Tables 1 and 2). The residual $V$ band image shows a number of structures. In particular, to the SW of NGC 3311 around the galaxy's major axis at PA $=212^{\circ}$, in a $\sim 90^{\circ}$-wide cone and radial range $40^{\prime \prime}<R<80^{\prime \prime}$, we see (unphysical) negative residuals of about 260 ADU per pixel with respect to the sky counts measured in the "empty" eastern boundary of the VLT/FORS1 image. This negative residual indicates that NGC 3311 is fainter than the 2D Sersic model there. This negative residual comes from fitting an azimuthally averaged model to an intrinsically asymmetric light distribution; we saw previously in Sect. 3.2 that the galaxy is brighter in the NE quadrant than in the other three quadrants.
Table 2. Parameters for the 2D GALFIT fit models of NGC 3311.

\begin{tabular}{lccc}
\hline \hline Parameter & $\begin{array}{c}V \text { band } \\
\text { FORS1 }\end{array}$ & $\begin{array}{c}V \text { band } \\
\text { FORS1 }\end{array}$ & $\begin{array}{c}V \text { band } \\
\text { FORS1 } \\
\text { max. symmetr. }\end{array}$ \\
\hline Comp. type & Sersic & Sersic & Sersic \\
$m_{\text {tot }}{ }^{*}$ & $9.7 \pm 0.01$ & $10.1 \pm 0.01$ & 9.52 \\
$R_{\mathrm{e}}{ }^{*}$ & $270.6 \pm 3$. ". & $198^{\prime \prime} 8 \pm 2.2$ & $850^{\prime \prime} \pm 50^{\prime \prime}$ \\
$n$ & $5.0 \pm 0.02$ & $4.8 \pm 0.02$ & $10.5 \pm 0.1$ \\
$b / a$ & 0.93 & 0.93 & 0.93 \\
PA & $38 \pm 0.5$ & 32 & 32 \\
mask & "cmask" & "allmask" & "allmask" \\
\hline
\end{tabular}

Notes. ${ }^{(*)}$ The $m_{\mathrm{tot}}$ and $R_{\mathrm{e}}$ values computed from the GALFIT fit model for NGC 3311 are respectively brighter and larger than those computed in Sect. 3.1 from the $V$ band light growth curve, because the latter is measured only out to $71^{\prime \prime} .5$, while the $2 \mathrm{D}$ model is fit to the whole $6.8 \times$ 6.4 area.

We therefore investigated whether by masking the whole north-east quadrant of NGC 3311, the "symmetric" 2D model for the $V$ band light in NGC 3311 would be assigned a lower value for the Sersic index $n$, and thus the problem of negative residuals at $\mathrm{PA}=212^{\circ}$ be reduced. We defined a new mask that additionally covers most of the NE quadrant in the NGC 3311 halo: this is shown in the right panel of Fig. 1 and is identified as "allmask". When the light excess is masked, the GALFIT 2D fit to the $V$ band FORS1 image returns a Sersic index $n_{V}=$ 4.8 and $R_{\mathrm{e}}=198^{\prime \prime} .8$ for NGC 3311. The GALFIT model of NGC 3309 and NGC 3311, obtained using "allmask" with the VLT/FORS1 data, and the residual image (difference between image and model) are shown in Fig. 9. Parameters are given in Tables 1 and 2.

The residual image still shows negative values in the same region in a $\sim 90^{\circ}$-wide cone around $\mathrm{PA}=212^{\circ}$ for the radial range $40^{\prime \prime}<R<80^{\prime \prime}$, but the level is now reduced to $110 \mathrm{ADU}$ per pixel, compared to $260 \mathrm{ADU}$ per pixel in the "cmask" residual image. Therefore, the extra light in the NE of NGC 3311 must extend slightly further than the masked area.

In addition, the residual image shows several positive valued structures that correspond to additional light in the real image with respect to the 2D models of NGC 3311 and NGC 3309, at a level of 200-500 ADU per pixel relative to sky. These structures are quantitatively discussed in greater detail in Sects. 4.2 and 4.3 


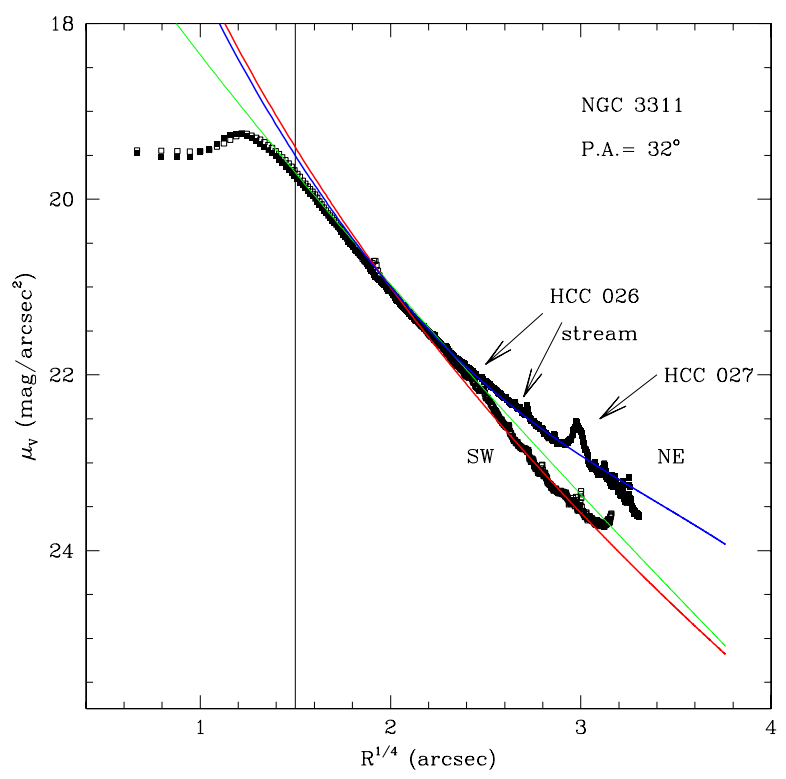

Fig. 10. Folded 1D surface brightness profile along the major axis of NGC 3311 plotted as function of $R^{1 / 4}$. Open symbols show values along $\mathrm{PA}=212^{\circ}(\mathrm{SW})$, full symbols those along $\mathrm{PA}=32^{\circ}(\mathrm{NE}$ of the galaxy center). The green continuous line shows the extracted 1D major-axis profile of the Sersic "allmask" model for the $V$-band FORS1 data described in Sect. 4.1. The red continuum line is the major axis profile for the light centered on NGC 3311 ("maximal" symmetric model, MSM). The blue full line shows the 1D fit to the surface brightness profile along $\mathrm{PA}=32^{\circ}(\mathrm{NE})$ with a two-component model, consisting of the MSM plus a one-sided exponential profile. The arrows indicate the position of the stream around HCC 026 and the light at $R \simeq 80^{\prime \prime}$ associated with the dwarf galaxy HCC 027 , which falls on the extracted profile. See Sect. 4.2 for details.

in the context of a "maximal" symmetric model with which the negative residuals are removed. Briefly,

- in the outer regions, NGC 3311 is more luminous than the best-fit $2 \mathrm{D}$ model in a wide area covering the whole $\mathrm{NE}$ quadrant to the galaxy. Superposed on this envelope, we also see a prominent tidal stream around HCC 026, a dwarf galaxy in the Hydra I cluster core. The stream is outlined by the red dashed lines in Fig. 9 at a distance of $\sim 43^{\prime \prime}$ from the center of NGC 3311. The tidal tails around HCC 026 extend about $15 \mathrm{kpc}$ NW and $5 \mathrm{kpc}$ NE of the dwarf galaxy;

- the S0 galaxy HCC 007 situated 119" south of NGC 3311 appears to be embedded in an extended, thick tidal stream visible at faint surface brightness levels. Emerging from HCC 007, the stream circles the south part of the Hydra I core, encompasses both NGC 3311 and NGC 3309, and extends over at least $450^{\prime \prime}(\sim 110 \mathrm{kpc})$, as indicated by the dashed blue lines in Fig. 9. The eastern tail of HCC 007 appears to join the bright envelope to the NE of NGC 3311; as part of the envelope, it may continue considerably further to the north.

Figure 10 shows 1D surface brightness profiles extracted along the NE and SW sides of the major axis of NGC 3311, as illustrated in Fig. 9. For radii $5^{\prime \prime}<R<30^{\prime \prime}$, the extracted profiles are straight lines in the $R^{1 / 4}$ plot: deviations from a de Vaucouleurs profile become large at $R>30^{\prime \prime}$ on the NE side $\left(\mathrm{PA}=32^{\circ}\right)$, indicating that there is additional light there. The flattening of the surface brightness profile at $\mathrm{PA}=32^{\circ}$ in Fig. 10 with respect to the steeper profile at $\mathrm{PA}=212^{\circ}$ indicates that at radii larger than $30^{\prime \prime}$, the outer surface brightness contours shift towards the NE; i.e., the envelope is off-centered to the NE relative to the bright central region of NGC 3311. This confirms the asymmetries of the extracted surface brightness profiles discussed in Sect. 3 .

Figure 10 also explains the origin of the negative residuals in the "allmask" model: the surface brightness profile fitted by GALFIT (green line) falls slightly above the SW (PA = $212^{\circ}$ ) major axis profile because of the surface brightness drop around 30 arcsec, so that the symmetric 2D model built from this profile generates negative values in the residual image about $\mathrm{PA}=212^{\circ}$.

In the next section, we improve on this by constructing a "maximal" symmetric model for the light centered on NGC 3311. We then use this to construct a new 2D model and discuss the structures in the corresponding residual image in more detail.

\subsection{The asymmetric light distribution around NGC 3311: extended off-centered envelope and tidal tails}

We built a maximal symmetric model (MSM) for NGC 3311 in two steps. First, we fit the steep surface brightness profile towards SW, along PA $=212^{\circ}$, with a Sersic law. Second, we build a 2D GALFIT model with the Sersic parameters fixed by the 1D fit to the steep profile, which therefore leads to positive or null residuals everywhere in the NGC 3311 halo.

Figure 10 shows the $1 \mathrm{D}$ fit profile over plotted on the data (red line); the model fits the data well in the radial range $15^{\prime \prime}-80^{\prime \prime}$. The parameters are $n_{V}=10.5 \pm 0.1, R_{\mathrm{e}}=850^{\prime \prime} \pm 50^{\prime \prime}$, and $\mu_{\mathrm{e}}=28.07 \pm 0.1 \mathrm{mag} \operatorname{arcsec}^{-2}$ (see Table 2). We then used GALFIT with the mask "allmask" to generate a 2D MSM for NGC 3311, using the Sersic law with parameters set from the $1 \mathrm{D}$ best-fit and the major axis $\mathrm{PA}=32^{\circ}$ and axis ratio 0.93 from our previous 2D model fits. Then, keeping this component fixed, we used GALFIT again to determine new best-fit parameters for NGC 3309, matching a Sersic model to the remaining light. The results of this NGC 3309 fit are listed in Table 1. Adding both components, we obtained the MSM for the two bright galaxies NGC 3311 and NGC 3309. By construction, the residual image obtained as the difference between the $V$-band VLT/FORS1 image and this MSM should have only positive or null residual values around NGC 3311 (outside the central $R<15^{\prime \prime}$ containing dust and bright knots).

This residual image, displayed in Fig. 11, clearly shows the presence of an additional, extended envelope, off-centered towards the NE of the inner regions of NGC 3311. It is highlighted by the white dashed ellipse in Fig. 11. The residual image also shows the bright tail around HCC 026, and the broader tail around HCC 007, which connects with the off-centered envelope. These structures stand out more clearly in Fig. 11 than in the earlier Fig. 9 because with the MSM we no longer have negative residuals. We note that the "half-ring" morphology around NGC 3311 slightly inside the HCC 026 stream probably signals that a small fraction of the light in the "maximal" symmetric inner halo for $r<30^{\prime \prime}$ should be counted as part of the off-centered envelope, such that its surface brightness on top of NGC 3311 becomes a constant $\mu_{V}=23.4 \mathrm{mag} \operatorname{arcsec}^{-2}$. However, we have no independent way of determining this light fraction. The "maximal" symmetric model is constructed to ensure that the minimal fraction of light is placed in the off-centered envelope.

To quantify the asymmetry in the NGC 3311 halo, we also fit the NE surface brightness profile in Fig. $10\left(\mathrm{PA}=32^{\circ}\right)$ with a two-component model, consisting of a Sersic law with the same $n$ and $R_{\mathrm{e}}$ and an additional exponential profile for the 

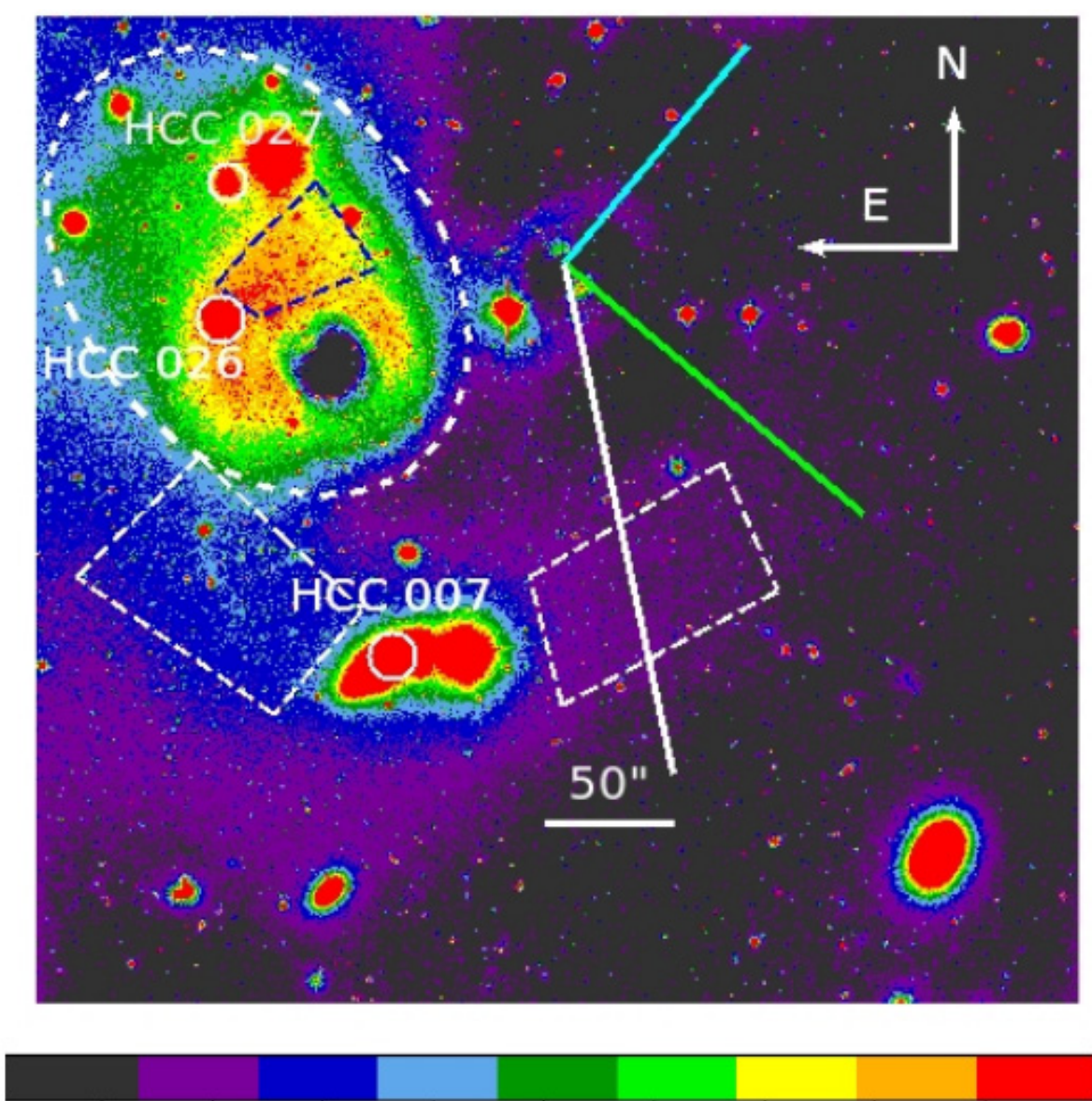

26.9325 .2624 .6324 .2423 .9523 .7223 .5323 .3723 .23
Fig. 11. Residual VLT/FORS1 $V$-band image with respect to the MSM for NGC 3311 and NGC 3309. The white ellipse indicates the extended halo off-centered towards the NE of the bright inner regions of NGC 3311 (black). The angular extent of this envelope as seen from the center of NGC 3311 is consistent with the opening angle of the additional light as shown by Figs. 7 and 8 . The most noticeable substructure on top of the extended envelope NE of NGC 3311 is the tidal stream associated with the dwarf galaxy HCC 026. Its NW part is indicated on the image with the blue dashed polygon. At larger radii, and at fainter surface brightness levels, we see a thicker tail around HCC 007; the luminosity in this tail is computed in the areas limited by the white polygons (see Sect. 4.3). The two small objects near the center of the NE polygon appear to be connected by a further small stream (see also Fig. 9). The FoV is 6! $8 \times 66^{\prime} 4$. Color levels are labeled with the corresponding surface brightness values. This image shows that in the region south of HCC 007 the background is flat, with a systematic E-W gradient across the image at less than $\sim 10$ ADU. The white, green, and blue lines from the center of NGC 3309 indicate the PAs at $=190^{\circ}, 230^{\circ}$, and $320^{\circ}$, respectively; see extracted profiles in Fig. 12. outer regions. The exponential profile is often a good approximation for the outer stellar envelopes of $\mathrm{cD}$ galaxies (Seigar et al. 2007). This combined model provides a good fit to the NE profile, which is also shown in Fig. 10 (blue line); the parameters are $n_{V}=10.5 \pm 0.1, R_{\mathrm{e}}=850^{\prime \prime} \pm 50^{\prime \prime}$, and $\mu_{\mathrm{e}}=28.2 \pm 0.1 \mathrm{mag} \operatorname{arcsec}^{-2}$ for the Sersic component, and $\mu_{0}=23.2 \pm 0.1 \mathrm{mag} \mathrm{arcsec}^{-2}$ and $r_{\mathrm{h}}=200^{\prime \prime} \pm 50^{\prime \prime}$ for the exponential component. The peak surface brightness of the offcentered envelope in the "maximal" symmetric halo model is $\mu_{0, V}=23.2 \mathrm{mag} \operatorname{arcsec}^{-2}$, as given by the exponential fit along $\mathrm{PA}=32^{\circ}$.

In the residual map, Fig. 11, the measured surface brightness at the position of the HCC 026 tail is also $\sim 23.2 \mathrm{mag} \mathrm{arcsec}^{-2}$. This is the combined surface brightness of the off-centered envelope and the tail, which are superposed along this LOS. The contribution of the HCC 026 tail within the polygon area limited by the blue dashed line in Fig. 11 is about 23\% (see Sect. 4.3). Hence, the average surface brightness of the tail around HCC 026 in the area enclosed by the blue dashed polygon in Fig. 11 is $\mu_{V}=24.8 \pm 0.2$ mag arcsec$^{-2}$.

The surface brightness of the tail around HCC 007 can be directly measured on the residual image. It is brighter on the NE side, where $\mu_{V}=24.4 \pm 0.5 \mathrm{mag} \operatorname{arcsec}^{-2}$, than on the NW side, where it is about one magnitude fainter. The brighter NE tail of HCC 007 is clearly a localized structure with a higher surface brightness than other areas at a similar distance from either NGC 3311 or NGC 3309. The central region of the brighter NE tail of HCC 007 contains two small objects that appear to be connected by a possible further small stream.
We can check with Fig. 12 that the NW tail of HCC 007 is not an artifact of residual halo light in NGC 3309. The upper panel of this figure shows that the minor axis profile of this galaxy $\left(\mathrm{PA}=320^{\circ}\right)$ is well-described by the fitted Sersic profile, whereas additional light is seen along $\mathrm{PA}=230^{\circ}$ and particularly along $\mathrm{PA}=190^{\circ}$ which goes through the brightest part of the NW tail close to HCC 007. However, it cannot be ruled out that this region contains some light from a very diffuse, low surface brightness structure around NGC 3311 and the cluster center.

Finally, we show that the morphologies of the substructures seen in the residual image in Fig. 11 are not due to systematic effects in the 2D modeling caused by uncertainties in the background estimates. This concern can be addressed by showing that these substructures are present in two independent datasets, the VLT/FORS1 and $2.2 \mathrm{~m} / \mathrm{WFI}$ images. We have already seen in Sect. 3.2 that the asymmetries in the extracted profiles along $\mathrm{PA}=32^{\circ}, 212^{\circ}$ are very similar in the two data sets; this confirms independently the presence of the off-centered envelope NE of NGC 3311. The lower panel of Fig. 12 compares the VLT/FORS1 and WFI surface brightness profiles through the fainter NW tail of $\mathrm{HCC} 007$ along PA $=190^{\circ}$ as seen from the center of NGC 3309. The light excess in the HCC 007 tail is clear in both data sets. Both results are independent of the slight residual gradient described in Sect. 3.2, because this is essentially perpendicular to $\mathrm{PA}=32^{\circ}$ and $\mathrm{PA}=190^{\circ}$. We also carried out the 2D GALFIT modeling on the $2.2 \mathrm{~m} /$ WFI $V$ band image, using the 1D maximal model-fit parameters for NGC 3311, and the "allmask" mask shown in Fig. 2 to determine the parameters 


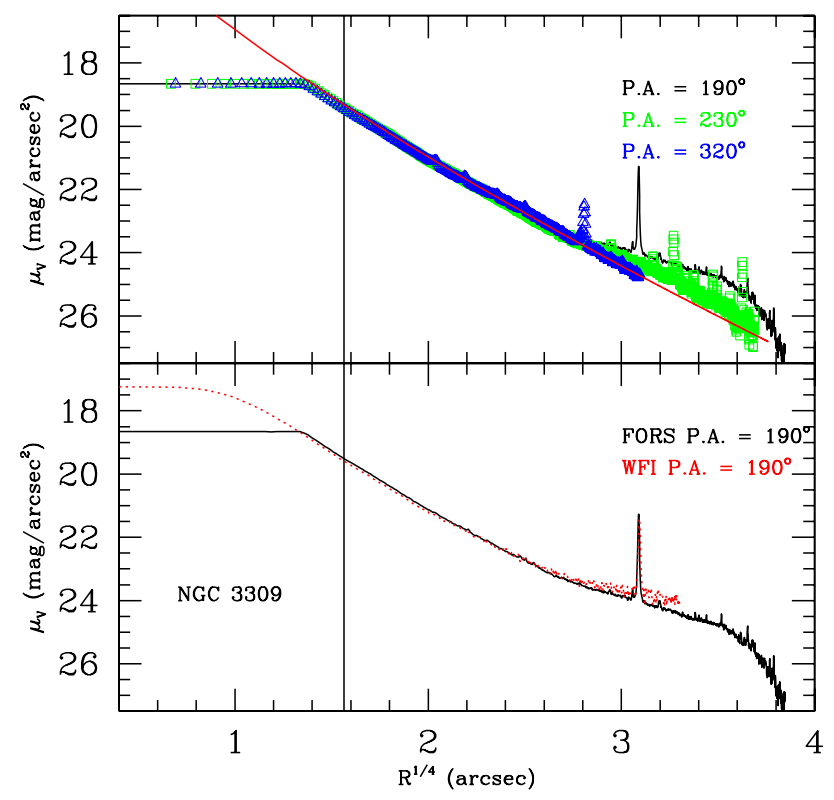

Fig. 12. Upper panel: surface brightness profiles extracted in $5^{\circ}$ wide cones along the major axis $\left(\mathrm{PA}=230^{\circ}\right.$, green squares), minor axis $\left(\mathrm{PA}=320^{\circ}\right.$, blue triangles $)$, and intermediate axis $\left(\mathrm{PA}=190^{\circ}\right.$, black line) of NGC 3309, which are plotted as a function of $R^{1 / 4}$. The PA at $190^{\circ}, 230^{\circ}$, and $320^{\circ}$ are indicated by the white, green, and blue lines from the center of NGC 3309 in Fig. 11. The red continuous line shows the extracted 1D major-axis profile for the 2D GALFIT "maximal" symmetric model (MSM) fit to the light of NGC 3309. While this is a good fit to the minor axis profile at large radii, the profiles along $\mathrm{PA}=230^{\circ}$ and $\mathrm{PA}=190^{\circ}$ show additional light, as they go through the brightest part of the HCC $007 \mathrm{NW}$ stream. Lower panel: surface brightness profile derived from VLT/FORS1 (black line) and 2.2/WFI (red line) $V$ band images along $\mathrm{PA}=190^{\circ}$.

of NGC 3309. The residual $V$-band $2.2 \mathrm{~m} / \mathrm{WFI}$ image has a lower $\mathrm{S} / \mathrm{N}$ than that based on the VLT/FORS1 data. Nonetheless, both elongated streams around HCC 026 and HCC 007 are independently confirmed. In the case of $\mathrm{HCC}$ 007, only the brighter NE tail is visible in the residual image.

\subsection{Luminosities of the substructures in the Hydra I core}

We then computed the total luminosity of the off-centered envelope from the flux within the elliptical aperture shown in Fig. 11. We masked stars, dwarf galaxies, and a circular region with $R=$ $25^{\prime \prime}$ centered on NGC 3311 . The resulting luminosity in the offcentered envelope is $L_{V, \mathrm{NE}, \text { env }}=1.2 \times 10^{10}\left( \pm 6.0 \times 10^{8}\right) L_{\odot}^{1}$, using a distance of $50 \mathrm{Mpc}$ for the Hydra I cluster. This estimate does not include the luminosity of the MSM halo in this region, but it does include the light in the stream around HCC 026, which is seen in addition to the exponential component in Fig. 10.

For comparison, we computed the luminosity of the MSM i) within the same elliptical aperture; and ii) in the circular annulus between $R=25^{\prime \prime}$ and $120^{\prime \prime}$, by integrating the flux of the GALFIT model output image over the respective areas. The luminosities of the MSM model evaluated in these two regions are $L_{V, \mathrm{NE} \text {, halo }}=2.3 \times 10^{10}\left( \pm 6.0 \times 10^{8}\right) L_{\odot}$ and $L_{V \text {,halo }}=$ $7.5 \times 10^{10}\left( \pm 2.0 \times 10^{9}\right) L_{\odot}$. Thus, within the area limited by the elliptical aperture in Fig. 11, the luminosity in the off-centered envelope amounts to $50 \%$ of the light in the MSM, while it

\footnotetext{
1 The $1-\sigma$ error in the luminosity was estimated by integrating the sky rms per pixel over the area enclosed by the elliptical aperture.
}

is only $15 \%$ of the total luminosity of the MSM integrated between $25^{\prime \prime}$ and $120^{\prime \prime}$.

To estimate the luminosity of the HCC 026 tail, we carried out surface photometry in a polygon designed to include most of the NW half of the stream; it is constructed with the IRAF POLYMARK and POLYPHOT tasks and is shown by the blue dashed polygon in Fig. 11. The polygon defined for the HCC 026 tail is at an average distance of 53". From the luminosity measured in this polygon, we subtracted the contribution of the smooth envelope in the same area, estimated from the average residual surface brightness on both sides of the stream. This gives us a differential measurement of the luminosity of the HCC 026 tail in the polygon. The resulting luminosity of the NW part of the stream is $L_{V, \mathrm{NW} \text { streamHCC } 026}=$ $4.8 \times 10^{8}\left( \pm 8 \times 10^{7}\right) L_{\odot}$, which is equivalent to an apparent magnitude of $m_{V \text {,streamHCC } 026}=16.6$. We find that the NW stream of HCC 026 contributes about $\sim 15 \%$ of the combined surface brightness of the MSM halo and off-centered envelope at the stream position (see also Fig. 10), which is consistent with the estimate obtained independently of the stellar population analysis in Coccato et al. (2011a). The luminosity in the southern part of the HCC 026 stream is more difficult to disentangle from the diffuse halo, but is comparable. It is seen in Fig. 8 as an excess of surface brightness of $\sim 0.1$ mag over $\sim 15^{\prime \prime}$ in the east profile over that on the west side. The total stream luminosity is clearly several times higher than the total luminosity of the dwarf galaxy HCC 026 itself, $L_{V, \mathrm{HCC} 026}=1.5 \times 10^{8} L_{\odot}($ Misgeld et al. 2008), and if the stream is physically related to HCC 026, a large part of the luminosity of this galaxy has already been tidally dissolved!

In a similar way, we computed the light in the tail emerging from HCC 007 . We defined two polygons shown by the white dashed boxes in Fig. $11 \mathrm{NE}$ and NW of HCC 007 and carried out the photometry with the POLYPHOT task. The polygons defined for the HCC 007 tails are at average distances of 120" and 173", where the surface brightnesses in the stream are 24.4 and 25.4 mag arcsec ${ }^{-2}$, and those of the "maximal" Sersic model are 24.3 and $24.8 \mathrm{mag} \operatorname{arcsec}^{-2}$. The apparent $V$ band magnitudes in the two polygons are $m_{v}=14.9$ and 16.3 for the NE and NW sides, respectively. At a distance of $50 \mathrm{Mpc}$, the combined luminosity in the two polygons on the HCC 007 tails is therefore $L_{V \text {,streamHCC } 007}=2.95 \times 10^{9}\left( \pm 5 \times 10^{8}\right) L_{\odot}$. For comparison, the $V$ band luminosity of HCC 007 inferred from the $V_{0}$ magnitude measured in Misgeld et al. (2008), $V_{0}=$ $14.18 \pm 0.01$, is $L_{V, \mathrm{HCC} 007}=4.5 \times 10^{9} L_{\odot}$. Thus, the ratio of the luminosity in the tail polygons to that of the S0 galaxy HCC 007 is $L_{V, \text { streamHCC } 007} / L_{\mathrm{HCC} 007}=0.66$. This is obviously a lower limit; in particular if the NE tail extends into the off-centered halo of NGC 3311, its luminosity may be substantially larger than estimated with the NE polygon. Thus, at least $40 \%$ of the original luminosity of HCC 007 has already been dissolved from the galaxy.

In summary, the extensive photometric analysis of our $V$ band images provides strong quantitative evidence of an extended envelope around NGC 3311 off-centered towards the NE, and for luminous streams around HCC 026 and HCC 007. We now wish to establish the kinematic association of these diffuse structures with their galaxies, with the goal of understanding their origin.

\section{Long-slit spectroscopy for the NGC 3311 outer halo and the tidal stream of HCC 026}

We first investigate whether the off-centered, extended envelope around NGC 3311 is associated with any clear signatures in the 
kinematics obtained from deep long-slit spectra. In Sect. 5.1, we discuss the mean LOS velocity and velocity dispersion profiles with respect to the center of NGC 3311. We then describe the link between kinematic and surface brightness features in order to constrain the dynamics of the inner galaxy and the outer halo. In Sect. 5.2, we seek spectroscopic evidence of the tidal tails emerging from HCC 026.

\subsection{Kinematic signatures of the off-centered envelope around NGC 3311}

In Ventimiglia et al. (2010), we showed that the halo around NGC 3311 for $R>15^{\prime \prime}$ is dynamically hot. The kinematic data for NGC 3311 show an extremely rapid rise from galaxytypical values of $\sigma_{\mathrm{LOS}}\left(R<15^{\prime \prime}\right) \simeq 170 \mathrm{~km} \mathrm{~s}^{-1}$ to velocity dispersions $\sigma_{\text {LOS }}\left(50^{\prime \prime}\right)>400 \mathrm{~km} \mathrm{~s}^{-1}$ dominated by the cluster potential. This study was based on Gemini long-slit spectra along $\mathrm{PA}=63^{\circ}$, and deep VLT/FORS2 long-slit spectra centered on the dwarf galaxy HCC 026 with a PA $=142^{\circ}$. More recently, the VLT/FORS 1 spectra along PA $=29^{\circ}$ published by Richtler et al. (2011) confirmed the large outer velocity dispersion values and the asymmetry of the $V_{\text {LOS }}$ profiles, but also showed deviations between measurements at different PA.

The mean LOS velocity $V_{\mathrm{LOS}}(R)$ profiles along both PAs increase outwards from NGC 3311 by $\sim 100 \mathrm{~km} \mathrm{~s}^{-1}$ to the NE, while they remain close to the galaxy systemic velocity $V_{\mathrm{NGC} 3311}(R)=3800 \mathrm{~km} \mathrm{~s}^{-1}$ to the SW. Thus, Ventimiglia et al. (2010) concluded that the NE outer halo has slightly more redshifted mean LOS velocities, $V_{\text {halo }} \simeq 3900 \mathrm{~km} \mathrm{~s}^{-1}$, and is offset by $\sim+100 \mathrm{~km} \mathrm{~s}^{-1}$ relative to the NGC 3311 center. The photometric evidence of an offset halo component to the NE of NGC 3311 shown in Fig. 11 suggests that the stars in the offset halo could be responsible for the shift in LOS velocities.

Building on the photometric model derived in Sect. 4.2, we can check whether either $\sigma_{\mathrm{LOS}}(R)$ and/or $V_{\mathrm{LOS}}(R)$ increase at positions where the surface brightness of the extended offset envelope is comparable to or brighter than the surface brightness of the MSM for NGC 3311. In Fig. 13, we illustrate this analysis, comparing kinematic and surface brightness profiles along the galaxy's approximate major axis $\left(\mathrm{PA}=29^{\circ}\right.$, and $\mathrm{PA}=32^{\circ}$, respectively). Both $V_{\mathrm{LOS}}$ and $\sigma_{\mathrm{LOS}}$ increase as the surface brightness of the offset envelope increases from $R \simeq 15^{\prime \prime}$ outwards. In particular, the $V_{\text {LOS }}$ values are more red-shifted on the NE side of the galaxy center than either at the center or in the SW region. However, this asymmetry peaks at $R \simeq 30^{\prime \prime}$ and does not closely follow the estimated relative surface brightness contribution of the off-centered envelope.

The outermost measurement from Ventimiglia et al. (2010) of $V_{\mathrm{LOS}}=3835 \mathrm{~km} \mathrm{~s}^{-1}$ and $\sigma_{\mathrm{LOS}}=429 \mathrm{~km} \mathrm{~s}^{-1}$ at $R=100^{\prime \prime}$ is relevant to this discussion. These values are measured for a $1 \mathrm{D}$ spectrum extracted from a region in the NGC 3311 halo that is only weakly affected by the off-centered envelope; the orangedashed line in Fig. 14 shows the location of this slit region on the image. These measurements therefore suggest that the symmetric halo is hot and approximately at the galaxy systemic velocity, and that the off-centered envelope is responsible for shifting the velocities measured in the NE quadrant of the NGC 3311 halo to more red-shifted values. We return to this issue in Sect. 6.

\subsection{Kinematical signatures of the tidal stream around HCC 026}

We now wish to confirm that the stream-like substructure in the diffuse envelope NE of NGC 3311 is physically associated with the dwarf galaxy HCC 026. The goal is to see whether we can

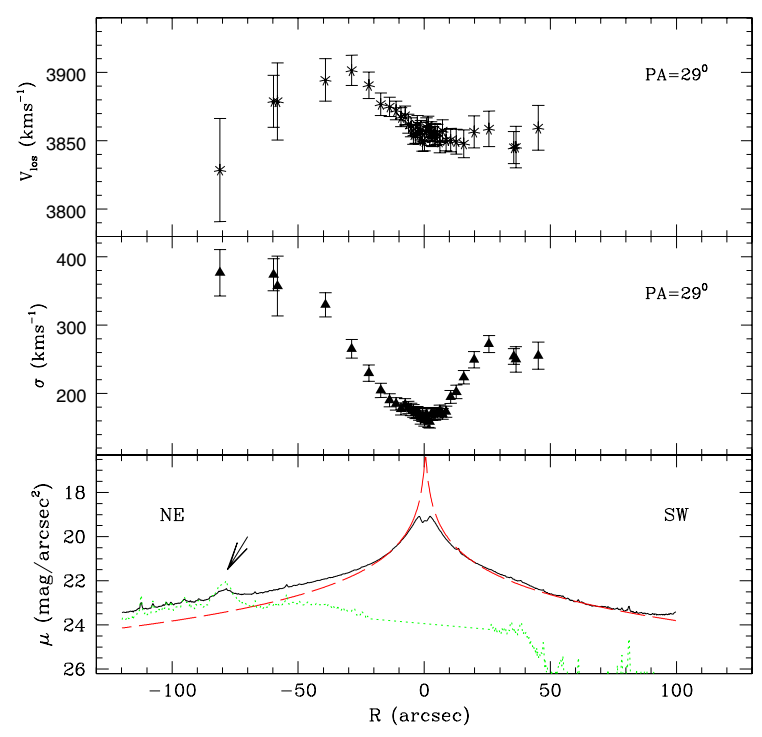

Fig. 13. Asymmetric kinematics and light distribution of the offcentered envelope around NGC 3311. Lower panel: the full black line shows the extracted surface brightness profile from the VLT/FORS1 $V$ band image along $\mathrm{PA}=32^{\circ}$, the red dashed line shows the Sersic profile $\left(n=10.5, R_{\mathrm{e}}=850^{\prime \prime}\right)$ of the MSM described in Sect. 4.2, and the green dashed line shows the contribution from the off-centered outer envelope along this PA The arrow indicates the dwarf galaxy HCC 027. Middle and upper panels: LOS velocity dispersion ( $\sigma_{\mathrm{LOS}}$, triangles) and velocity measurements ( $V_{\mathrm{LOS}}$, asterisks) along $\mathrm{PA}=29^{\circ}$ from Richtler et al. (2011) $\left(\mathrm{PA}=29^{\circ}\right.$ shown as $\left.R<0\right)$.

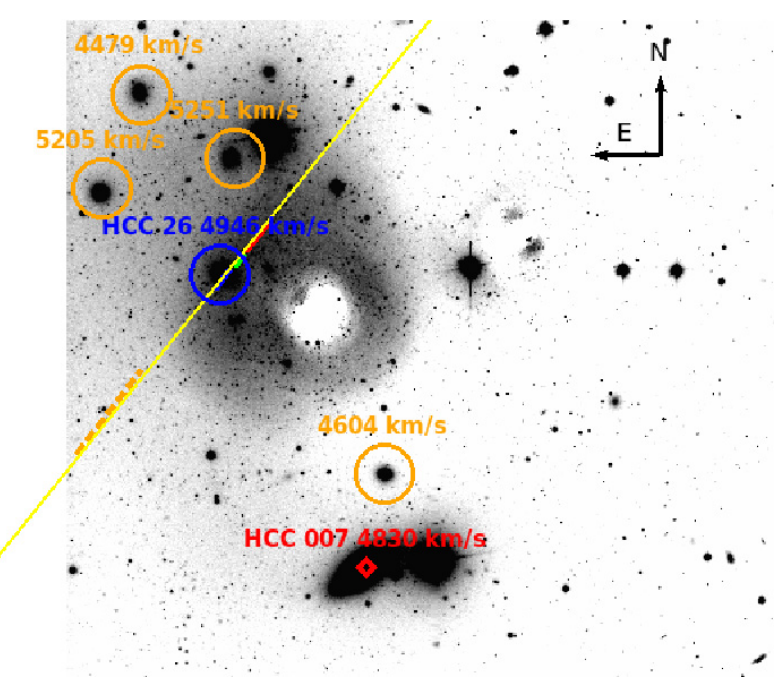

Fig. 14. Location of dwarf galaxies and long slit on the residual VLT/FORS1 $V$-band image from Fig. 11. Here the orange circles indicates the dwarf galaxies within $100 \mathrm{kpc}$ of the NGC 3311 center; their velocities are reported on the image. The blue circle shows the dwarf galaxy HCC 026, and the red diamond indicates HCC 007. The object to the right of HCC 007 is a foreground star. The yellow line centered on $\mathrm{HCC} 026$ at $\mathrm{PA}=142^{\circ}$ illustrates the position and orientation of the long slit used for the kinematic measurements in Ventimiglia et al. (2010) and in this paper. The orange-dashed section of the slit identifies the area where the $V_{\mathrm{LOS}}, \sigma$ measurements at $R=100^{\prime \prime}$ were taken (Ventimiglia et al. 2010); see Sect. 5.1. The red and green parts of the line depict the slit sections used to measure the mean velocity of the tidal stream. The FoV is $5 ! 1 \times 2 ! 8$; north is up and east to the left.

detect absorption line features in the spectra at the position of the tidal stream, in addition to the absorption lines from the stars in this part of the dynamically hot halo of NGC 3311. 
Table 3. Dwarf galaxies in the NGC 3311 field.

\begin{tabular}{ccccc}
\hline \hline Galaxy & $\begin{array}{c}\alpha(2000) \\
{[\mathrm{h}: \mathrm{m}: \mathrm{s}]}\end{array}$ & $\begin{array}{c}\delta(2000) \\
{\left[:^{\circ}::^{\prime \prime}\right]}\end{array}$ & $\begin{array}{c}M_{V} \\
{[\mathrm{mag}]}\end{array}$ & $\begin{array}{c}v \\
{\left[\mathrm{~km} \mathrm{~s}^{-1}\right]}\end{array}$ \\
\hline HCC 019 & $10: 36: 52.573$ & $-27: 32: 16.34$ & 16.91 & $5735 \pm 55$ \\
HCC 022 & $10: 36: 40.373$ & $-27: 32: 57.68$ & 18.23 & $4605 \pm 37$ \\
HCC 023 & $10: 36: 48.911$ & $-27: 30: 01.49$ & 18.07 & $4479 \pm 44$ \\
HCC 024 & $10: 36: 50.140$ & $-27: 30: 46.20$ & 17.75 & $5270 \pm 32$ \\
HCC 026 & $10: 36: 46.000$ & $-27: 31: 25.10$ & 17.87 & $4946 \pm 04^{*}$ \\
HCC 027 & $10: 36: 45.700$ & $-27: 30: 31.30$ & 18.48 & $5251 \pm 89$ \\
HCC 007 & $10: 36: 41.200$ & $-27: 33: 39.60$ & 14.18 & $4830 \pm 13$ \\
\hline
\end{tabular}

Notes. Magnitudes and LOS velocities are from Misgeld et al. (2008). ${ }^{(*)}$ Value from Ventimiglia (2011).

We use the deep long-slit spectroscopic observations of the NGC 3311 halo carried out by Ventimiglia et al. (2010) with FORS2 on VLT-UT1. These spectra extend over the wavelength range $4655-5955 \AA$ and include absorption lines from $\mathrm{H}_{\beta}, \mathrm{MgI}(\lambda \lambda 5167,5173,5184 \AA)$, and Fe I ( $\lambda \lambda 5270$, $5328 \AA$ ). They were acquired with a 6.8 arcmin long-slit of width 1.' 6 , and GRISM $1400+\mathrm{V}$, giving an instrumental dispersion of $0.64 \AA \mathrm{Apixel}^{-1}$ and a spectral resolution of $\sigma=90 \mathrm{~km} \mathrm{~s}^{-1}$. The long slit was centered on the dwarf galaxy HCC 026 at $\alpha=10^{\mathrm{h}} 36^{\mathrm{m}} 45.85^{\mathrm{s}}$ and $\delta=-27^{\mathrm{d}} 31^{\mathrm{m}} 24.2^{\mathrm{s}}$ (J2000), with a position angle $\mathrm{PA}=142^{\circ}$ so that is passes through the photometric stream. HCC 026 itself is seen in projection onto the NGC 3311 halo. The slit geometry is shown in Fig. 14. Eight exposures of $1800 \mathrm{~s}$ each were taken (in total $4 \mathrm{~h}$ ). In addition to the deep spectra, the standard G8III star HD 102070 and the spectrophotometric standard star EG 274 were also observed with the same set-up. The data reduction followed standard procedures as described in Ventimiglia et al. (2010). The final deep 2D spectrum was then used to construct radial profiles of the velocity dispersion and mean LOS velocity for the halo of NGC 3311 from measurements at various positions along the slit.

Here we wish to additionally detect in the spectrum the absorption lines of the stream superposed onto the main absorption features of the hot NGC 3311 halo. We expect these secondary absorption lines to have strengths of about $15 \%$ of those for the NGC 3311 halo, based on the photometry carried out in Sect. 4.2 (see Figs. 10, 11, and 13). If the subcomponent projected onto the outer halo is a tidal stream emerging from HCC 026, we expect its absorption lines to occur at significantly different recession velocities from those of the NGC 3311 diffuse halo $\left(V_{\text {halo }}=3921 \mathrm{~km} \mathrm{~s}^{-1}\right)$. This is because all of the dwarf galaxies seen at this location, including HCC 026 , have recession velocities of order $5000 \mathrm{~km} \mathrm{~s}^{-1}$ (see Table 3 and Ventimiglia et al. 2011).

We first extracted the light profile along the slit and identified the regions where the continuum is bright enough to provide a suitable $\mathrm{S} / \mathrm{N}$ for the kinematic measurements. We were able to identify only two such regions to the north-west of HCC 026: region I - 7" wide (30 pixels) - and region II - 18" wide (75 pixels). These are indicated by the green and red slit sections in Figs. 14 and 15. We co-added all the flux in each of these regions to reach $S / N \simeq 20$ in the continuum for the final one-dimensional (1D) extracted spectra. We must be aware that co-adding all the signal from an extended portion of the slit causes a broadening of the absorption lines, but this does not affect our goal, which is the detection of a secondary absorption-line component at a different LOS velocity from that associated with the hot halo component.

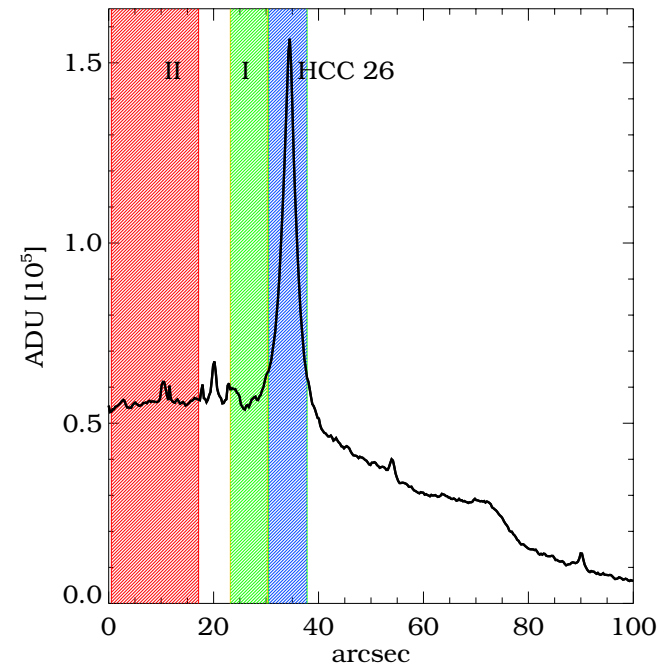

Fig. 15. Light profile along the slit centered on HCC 026, with the different color-shaded areas indicating the slit sections used for the extraction of the 1D spectra of HCC 026 (blue) and the tidal stream (green and red). Left is SE of HCC 026, right is NW.

To measure the stellar kinematics, we restricted ourselves to the central wavelength range $4800 \AA<\lambda<5800 \AA$ of the extracted 1D spectrum, and used the "penalized pixelfitting" method (PPXF Cappellari \& Emsellem 2004). In the PPXF method, stellar template stars from the MILES library (Sanchez-Blazquez et al. 2007) were combined to fit the 1D spectrum while simultaneously determining the mean LOS velocity and velocity dispersion from the absorption lines. From the best PPFX fit to the co-added spectrum, we obtained values for these kinematic parameters that are consistent with the halo kinematics measured by Ventimiglia et al. (2010), as well as a best-fit stellar template for the halo of NGC 3311.

From the photometry carried out in Sect. 4.2, the luminosity from the tidal stream amounts to $\sim 15 \%$ of the total light in the region sampled by this spectrum. Attempting a direct spectral decomposition of the stellar halo of NGC 3311 and the light excess as in Coccato et al. (2011b) was unsuccessful. To be able to detect the weak kinematic signal from the excess of light, we therefore needed to subtract the main halo contribution. We achieved this by taking the best-fit stellar template spectrum obtained by PPFX for the total extracted science spectrum, multiplying it by a suitable fraction $(0.85)$ and subtracting it off the extracted spectrum. This procedure when applied to the 1D spectra I and II leaves the residual spectra shown in Fig. 16. 


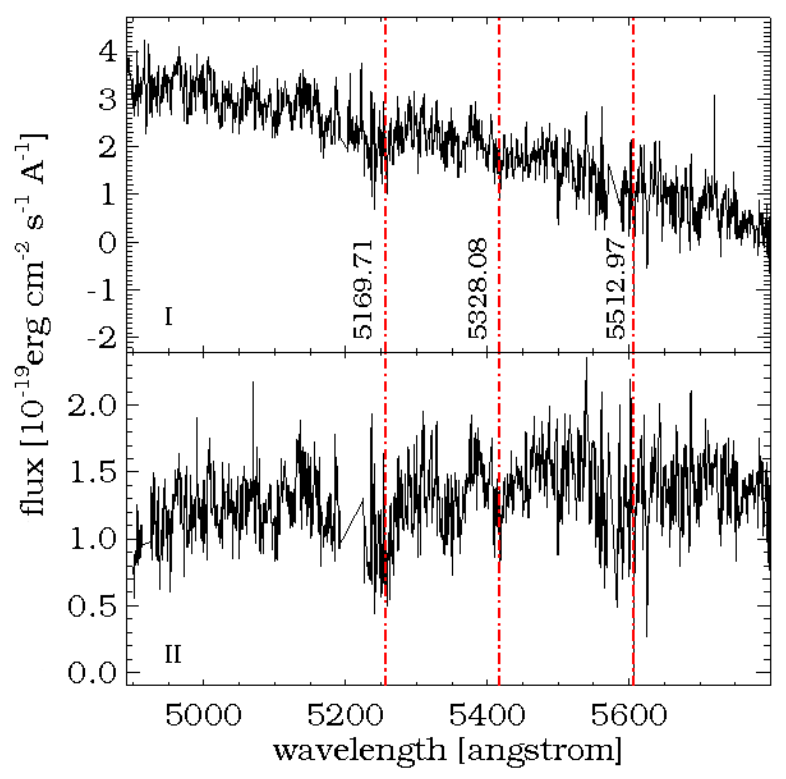

Fig. 16. One dimensional wavelength and flux-calibrated residual spectra I (upper panel) and II (lower panel), extracted at the position of the light excess. These 1D spectra are obtained by subtracting a template model of the NGC 3311 halo from the extracted 1D spectra. The vertical dash-dotted lines indicate the locations of the main absorption features whose rest-frame wavelengths are also reported on the figure.

The $\mathrm{S} / \mathrm{N}$ of the residual spectra is clearly not high enough for a direct pixel fitting, although the main absorption features are readily identified, and we therefore had to use a different approach. We used the RV.FXCOR task in IRAF to identify the velocity components in the residual spectrum; this task implements the Fourier cross-correlation technique of Tonry \& Davis (1979). The upper panels of Fig. 17 show the Fourier crosscorrelation functions computed with RV.FXCOR between the residual 1D spectra I and II and the extracted 1D spectrum of the star HD 102070. All spectra have their continuum fitted and subtracted-off, and only the wavelength interval $4800 \AA<\lambda<$ $5800 \AA$ is used.

In the lower panels of Fig. 17, the region centered around the two strongest correlation peaks is enlarged. This reveals the presence of two components at different velocities in both spectra: the strongest peak is found at $5054 \mathrm{~km} \mathrm{~s}^{-1}, 5010 \mathrm{~km} \mathrm{~s}^{-1}$, and a second peak is detected at $3931 \mathrm{~km} \mathrm{~s}^{-1}, 3889 \mathrm{~km} \mathrm{~s}^{-1}$, for spectra I and II respectively. The error in each velocity measurement is $\pm 55 \mathrm{~km} \mathrm{~s}^{-1}$. The estimated rms noise in the velocity range $2000-8000 \mathrm{~km} \mathrm{~s}^{-1}$ in the two lower panels of Fig. 17 is $\simeq 0.066$ and $\simeq 0.038$. The height of both peaks in the crosscorrelation spectrum in each panel is therefore $\sim 2$ times the amplitude of the noise; because the two panels are for two independent spectra, we therefore judge these signals to be significant. The bluer velocity peaks correspond to a component from the extended halo light that is still present in the residual spectra at an average LOS velocity of $3905 \mathrm{~km} \mathrm{~s}^{-1}$ (see also Sect. 5.1). The peaks at more redshifted LOS velocities indeed provide evidence of a second component at a relative velocity of $\Delta V \simeq$ $+1200 \mathrm{~km} \mathrm{~s}^{-1}$ with respect to the systemic velocity of NGC 3311 $\left(V_{\mathrm{NGC}} 3311=3800 \mathrm{~km} \mathrm{~s}^{-1}\right)$, and $\Delta V \simeq+1100 \mathrm{~km} \mathrm{~s}^{-1}$ relative to the halo $\left(V_{\text {halo }}=3921 \mathrm{~km} \mathrm{~s}^{-1}\right)$. The average LOS velocity obtained by combining the measurements from spectra I and II is $5032 \pm 38 \mathrm{~km} \mathrm{~s}^{-1}$. For comparison, the LOS velocity of HCC 026 is $4946 \pm 4 \mathrm{~km} \mathrm{~s}^{-1}$ (Ventimiglia et al. 2010).
In summary, the stars in the stream NE of HCC 026 have a LOS velocity very similar to that of HCC 026 itself but $\Delta V \simeq$ $+1200 \mathrm{~km} \mathrm{~s}^{-1}$ different from the systemic velocity of NGC 3311 . This is a necessary condition for the stream around HCC 026 being a tidal stream, but could also be consistent with the stream stars being part of a shell that was stripped from a larger galaxy together with HCC 026. However, combining our kinematic results with the result of Coccato et al. (2011a) that the stellar population at the NW stream position is more metal poor than in the surrounding outer halo, is consistent with the superposed stream stars having a similar metallicity to the dwarf galaxy HCC 026 itself, strongly implies that the stars in the stream were indeed tidally stripped from HCC 026.

\section{Correspondence between surface brightness components and kinematical structures measured with planetary nebulae, and the velocities along the $\mathrm{HCC} 007$ tail}

To begin with, we summarize the results from observations of the PN kinematics in the Hydra I cluster core, in order to see how they may help in understanding the physical structures identified in the photometry. In Ventimiglia et al. (2011), we measured LOS velocities for $56 \mathrm{PNs}$ in a $6.8^{\prime} \times 66^{\prime} 8$ field covering the central $(100 \mathrm{kpc})^{2}$ of the Hydra I cluster, using multi-slit imaging spectroscopy (MSIS, Gerhard et al. 2005; Arnaboldi et al. 2007). We identified different velocity components in the PN LOSVD and described their spatial distributions. In brief, we found:

- A broad, asymmetric central velocity component in the PNs LOSVD, peaked at $\sim 3100 \mathrm{~km} \mathrm{~s}^{-1}$ and with $\sigma \simeq$ $500 \mathrm{~km} \mathrm{~s}^{-1}$. The spatial distribution of these PNs has significant substructure, depending on the LOS velocity. It is unclear whether this component traces the symmetric halo around NGC 3311.

- A minor blue-shifted velocity component, centered at $\sim 1800 \mathrm{~km} \mathrm{~s}^{-1}$, whose PNs have an elongated distribution along the north/south direction. There is no galaxy with a similar velocity inside the $6.8^{\prime} \times 6$ ' $^{\prime} 8$ field centered on NGC 3311, but two of these galaxies are located near the perimeter of the field. The association of these with the blue-shifted PNs however is possible but unclear.

- A narrow red-shifted velocity component, around $V_{\mathrm{LOS}} \simeq$ $5000 \mathrm{~km} \mathrm{~s}^{-1}$. These red-shifted PNs show a concentration in the NE quadrant of NGC 3311, and are correlated spatially and in terms of the LOS velocity with a group of dwarf galaxies, which are observed within $(100 \mathrm{kpc})^{2}$ of NGC 3311.

In Fig. 18, we plot the PNs associated with the red-shifted peak of the PN LOSVD (Ventimiglia et al. 2011) in the residual VLT/FORS1 $V$ band image with respect to the MSM for NGC 3311. This shows that a major fraction of these redshifted PNs are concentrated in the NE quadrant where the offset halo is found. We count 10 PNs superposed on the offset envelope. Their average LOS velocity is $V_{\text {redPNs,LOS }}=5095 \mathrm{~km} \mathrm{~s}^{-1}$ and $\sigma_{\text {redPNs,LOS }}=521 \mathrm{~km} \mathrm{~s}^{-1}$. The average LOS velocity is similar to that of HCC $026\left(V_{\mathrm{HCC} 026}=4946 \mathrm{~km} \mathrm{~s}^{-1}\right)$ and to the value for the second component measured in Sect. $5.2\left(5032 \mathrm{~km} \mathrm{~s}^{-1}\right.$ average). With a relative offset of $1200 \mathrm{~km} \mathrm{~s}^{-1}$ from NGC 3311 and a contribution of $\simeq 15 \%$ to the surface brightness, the shift in the predicted mean velocity would be of order $180 \mathrm{~km} \mathrm{~s}^{-1}$, which is consistent with the measured value. However, we found in Sect. 4.3 that the off-centered envelope contributes $\sim 30 \%$ 

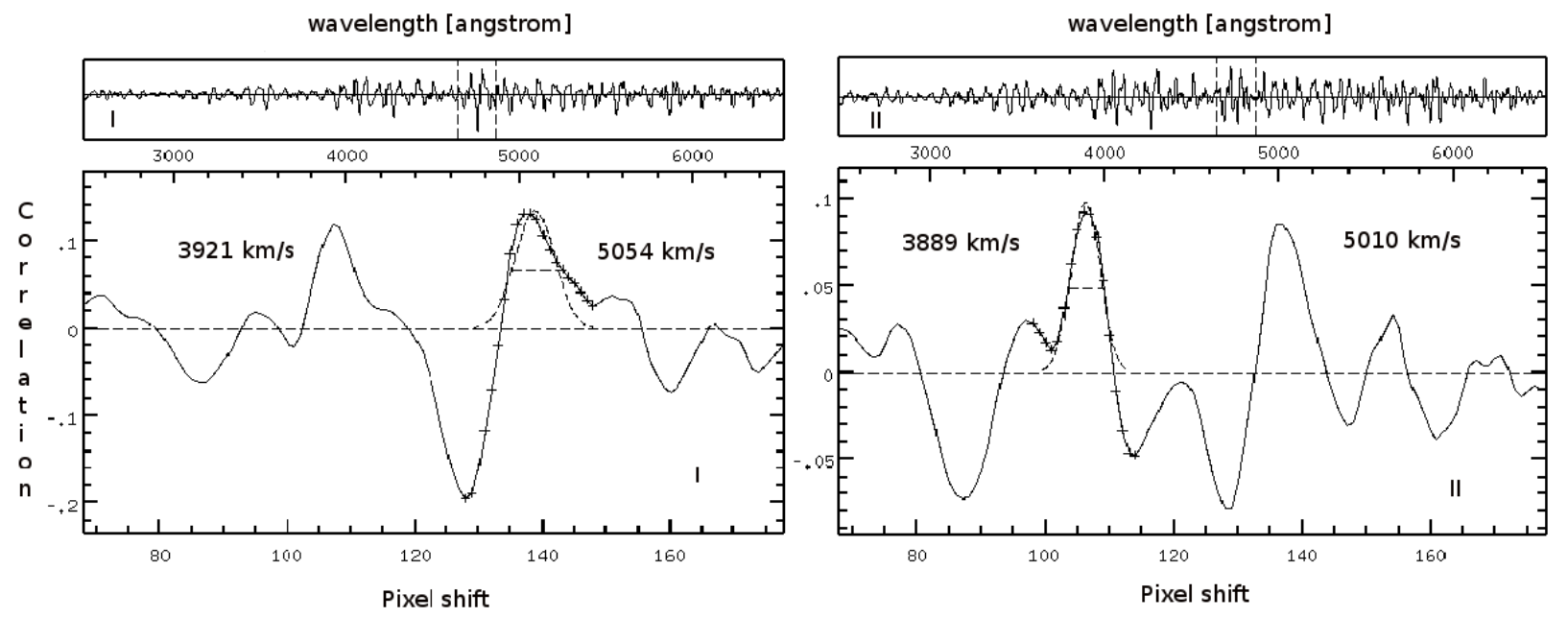

Fig. 17. Cross-correlation functions used for kinematic measurements in two slit sections. Upper panels: Fourier cross-correlation functions computed between the residual (excess) spectra and the spectrum of the G dwarf star HD 102070 for spectrum I (left) and II (right). Lower panels: enlarged view of the Fourier cross-correlation function peaks. The double peaks in both Fourier cross-correlation functions indicate the presence of two sets of absorption-line features at different velocities in the residual spectra. The more red-shifted peaks in the Fourier cross-correlation are at velocities $V_{\mathrm{LOS}, 1}=5054,5010( \pm 55) \mathrm{km} \mathrm{s}^{-1}$, and the secondary bluer peaks are at $V_{\mathrm{LOS}, 2}=3921,3889( \pm 55) \mathrm{km} \mathrm{s}^{-1}$.

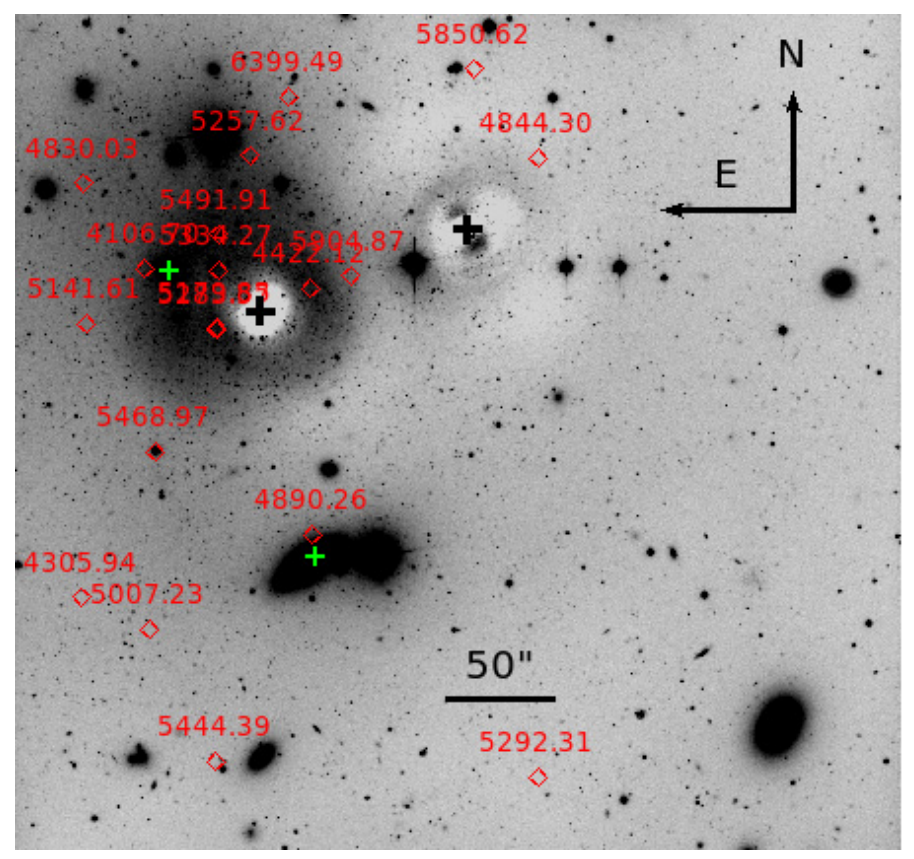

Fig. 18. Positions and velocities of PNs (red diamonds, labels indicate the PNs' $V_{\mathrm{LOS}}$ in $\mathrm{km} \mathrm{s}^{-1}$ ) associated with the red peak at $5000 \mathrm{~km} \mathrm{~s}^{-1}$ in the PN LOSVD of the Hydra I cluster, from Ventimiglia et al. (2011). The red peak PNs are superposed on the residual $V$-band image, which shows the substructures in the diffuse light in the Hydra I cluster core. The black crosses indicate the position of NGC 3311 (center) and NGC 3309 (upper right), respectively. The green crosses indicate HCC 026 and HCC 007. The FoV is 6 ${ }^{\prime} 8 \times 6$ 6 $^{\prime} 4$.

of the surface brightness in the NE region, and this value is consistent with the value indicated at the HCC 026 position in Figs. 10 and 13. Thus, while from their spatial location the red-shifted PNs appear to trace the off-centered envelope, it is unclear whether they trace all the stars in it.

Therefore, from the off-centered envelope's luminosity estimated in Sect. $4.3, L_{V, \text { NE, env }}=1.2 \times 10^{10}\left( \pm 6.0 \times 10^{8}\right) L_{\odot}$, we can derive an upper limit to the luminosity specific PN number $\alpha$ for this stellar population. The measured value is $\alpha=1 / 1.2 \times$ $10^{9} \mathrm{PN} L_{\odot}^{-1}$; once we correct this number for the limiting magnitude of these MSIS observations $\alpha_{\mathrm{TOT}}=82 \times \alpha$ (see Ventimiglia et al. 2011 for further details), we obtain $\log \left(\alpha_{\mathrm{TOT}}\right)=-7.18$. This value for $\alpha_{\mathrm{TOT}}$ is similar to that for the old stellar populations observed in the M31 bulge and S0 galaxies (Buzzoni et al. 2006).

For the symmetric halo of NGC 3311, the $\alpha$ parameter must be lower. Within $25^{\prime \prime}<R<120^{\prime \prime}$, we count 12 PNs from the central velocity component (Ventimiglia et al. 2011). If these trace the total luminosity of the symmetric halo, $L_{V \text {,halo }}=7.5 \times$ $10^{10}\left( \pm 2.0 \times 10^{9}\right) L_{\odot}$, the measured $\alpha=1 / 6.2 \times 10^{9} \mathrm{PN} L_{\odot}^{-1}$ and the corresponding $\log \left(\alpha_{\mathrm{TOT}}\right)$ is -7.87 , which is a factor of five lower than the value for the stellar population in the offset envelope. The LOSVD of the central PN component is quite axisymmetric, and very few PNs at the systemic velocity of NGC 3311 are found in the inner symmetric halo. Thus, the $\log \left(\alpha_{\mathrm{TOT}}\right)$ for this component may be even lower.

Superposed on the off-centered envelope, there are two tidal streams in the Hydra I core, which are shown in Figs. 9 and 11. In Sect. 5.2, the direct measurement of $V_{\mathrm{LOS}}$ of the stream around HCC 026 is $5032 \pm 38 \mathrm{~km} \mathrm{~s}^{-1}$, and the systemic velocities of HCC 026 and HCC 007 are $V_{\mathrm{HCC}} 026=4946 \mathrm{~km} \mathrm{~s}^{-1}$ and $V_{\mathrm{HCC} 007}=4830 \mathrm{~km} \mathrm{~s}^{-1}$, respectively. Four of the PNs plotted in Fig. 18 are consistent with being located on the NE HCC 007 stream (one is on the polygon used to estimate the luminosity). These indicate that there is a systematic velocity gradient along the stream, increasing from $4830 \mathrm{~km} \mathrm{~s}^{-1}$ east of the offcentered envelope to a maximum of $5470 \mathrm{~km} \mathrm{~s}^{-1}$ near the closest approach to NGC 3311, and decreasing again to $4890 \mathrm{~km} \mathrm{~s}^{-1}$ near HCC 007 itself.

Assuming that the stellar population in these streams is similar to that in the off-set envelope, we would expect $2 \pm 1 \mathrm{PN}$ in the polygon on the NE tail of HCC 007, and no PNs on the SE tail or on the stream around HCC 026. In Fig. 18, we count one PN associated with the corresponding part of the HCC 007 stream, which is consistent with the prediction. There is not enough light in the stream around HCC 026 to expect any PN to be detected within the limiting magnitude of the Ventimiglia et al. (2011) survey. 


\section{Discussion}

\subsection{The peculiar outer halo of NGC 3311}

Have we detected the outer halo or intracluster light? On the basis of $V$-band photometry out to $\sim 100^{\prime \prime}(\sim 25 \mathrm{kpc})$, the outer halo of NGC 3311 can be represented by a symmetric Sersic model with large $n \simeq 10$, and an additional off-centered component with centroid shifted by about $50^{\prime \prime}$ to the NE (Sect. 4). The offcentered component can be described by a (flatter) exponential profile. However, it is not the extended light profile that distinguishes this central cluster galaxy from other luminous elliptical galaxies, but its very steeply rising velocity-dispersion profile (VDP) $\sigma(R)$. From a central value of $\sim 170 \mathrm{~km} \mathrm{~s}^{-1}$, the VDP rises to $\sigma \simeq 230 \mathrm{~km} \mathrm{~s}^{-1}$ at $R=15^{\prime \prime} \simeq 3.7 \mathrm{kpc}$, and then on to $\sigma=300-450 \mathrm{~km} \mathrm{~s}^{-1}$ at $R=50^{\prime \prime} \simeq 12 \mathrm{kpc}$ (Ventimiglia et al. 2010; Richtler et al. 2011). The steep rise in the velocity dispersion profile corresponds to a steep increase in the enclosed mass. Within $R=20 \mathrm{kpc}$, the total dark matter mass inferred from X-ray observations is $\sim 10^{12} M_{\odot}$ (Hayakawa et al. 2004). Thus, NGC 3311 is located at the center of the dark matter cusp of the Hydra I cluster.

In the NW region dominated by the off-centered halo component, the velocity dispersion is particularly high $\left(\sim 450 \mathrm{~km} \mathrm{~s}^{-1}\right)$, at about $60 \%$ of the galaxy velocity dispersion in the cluster core (Ventimiglia et al. 2010). Its mean LOS velocity is also shifted by $\sim 100 \mathrm{~km} \mathrm{~s}^{-1}$ from that of NGC 3311. Many PNs located on the off-centered envelope move at LOS velocities of $+1200 \mathrm{~km} \mathrm{~s}^{-1}$ with respect to the NGC 3311 center (Ventimiglia et al. 2011), but the LOSVD of the "central PN component" of Ventimiglia et al. (2011) is also quite asymmetric. The off-centered envelope may therefore equally well be considered as part of the ICL in the cluster core; because of the steeply rising VDP, distinguishing outer halo from the ICL is difficult (see Dolag et al. 2010).

Are these dynamically hot outer halos in other BCGs? A galaxy with a dynamically hot outer halo similar to NGC 3311 is NGC 6166, the cD galaxy in the cluster A2199 (Kelson et al. 2002). In this system, the velocity dispersion first decreases from the central value of $300 \mathrm{~km} \mathrm{~s}^{-1}$ to $200 \mathrm{~km} \mathrm{~s}^{-1}$ within a few kiloparsecs, and then steadily rises to $660 \mathrm{~km} \mathrm{~s}^{-1}$ at a radius of $60 \mathrm{kpc}$, nearly reaching the velocity dispersion of the cluster $\left(\sigma_{\mathrm{A} 2199}=775 \pm 50 \mathrm{~km} \mathrm{~s}^{-1}\right)$. This is similar but less extreme than the case of NGC 3311 studied here, which does not show the central decrease. However, such dynamically hot outer halos in BCGs are rare: for instance for NGC 1399 in the Fornax cluster (McNeil et al. 2010), and both NGC 4874 and NGC 4989 in Coma (Coccato et al. 2010a) the outer VDPs are flat, while for M 87 in the Virgo cluster the VDP first rises out to 250" and then falls steeply (Doherty et al. 2009; Murphy et al. 2011).

Asymmetry in X-ray observations of the Hydra I cluster core - Independent evidence of asymmetries in the Hydra I cluster center comes from Chandra and XMM observations (Hayakawa et al. 2004, 2006). These authors report that there is extended emission $\sim 1.5$ in the direction NE of NGC 3311 relative to the iso-surface brightness contours further out, with an angular scale of about 1.5' (see Fig. 2 in Hayakawa et al. 2004; and Fig. 5 in Hayakawa et al. 2006). The $\mathrm{X}$-ray surface brightness maps show a morphology reminiscent of the off-centered halo in the stellar light distribution. The extended X-ray emission NE of NGC 3311 is also the brightest among several high-metallicity regions in the Hydra I cluster, and its associated gas mass is higher than that in the compact X-ray halo of NGC 3311. Hayakawa et al. (2004) interpreted this high-metallicity region 1.5 to the NE of NGC 3311 as the result of gas stripping of NGC 3311, which would imply that there is a relative motion of NGC 3311 with respect to the surrounding outer halo. It may be possible that the collision with the dark matter halo of the group including HCC 007 could have induced such a relative motion, an idea that requires further study.

Off-centered outer envelopes in BCGs - Separate outer components in BCGs are frequent; for a sample of 24 clusters Gonzalez et al. (2005) showed that a two-component fit to the light profiles of BCGs provides an improved match to the data, and that the two photometric components are misaligned in $60 \%$ of the sample. Gonzalez et al. (2005) also reported the case of Abell 1651, where the two components have different centers, with the outer component being off-centered by about $15 \mathrm{kpc}$ in linear distance. This is similar to the separation between the center of the off-centered envelope described in Sect. 4, and also the center of the extended X-ray emission, from the inner parts of NGC 3311.

Stellar population in the outer halo of NGC 3311 - Coccato et al. (2011a) measured the line strength indices for the stellar population in the NGC 3311 halo along a 6!.8 slit centered on HCC 026 and with PA $=142^{\circ}$. These measurements show the effect of the stars in the HCC 026 tail: in this region, the metallicity is lower, $[\mathrm{Fe} / \mathrm{H}]=-0.73 \pm 0.06$, compared to the average value for the outer halo away from the tail, where $[\mathrm{Fe} / \mathrm{H}]_{\text {halo }}-0.34 \pm 0.05$. The metallicity in the tail region is consistent with a $\sim 20 \%$ contribution of stars that have the same metallicity as HCC 026. Unfortunately, in the halo region away from the HCC 026 tail, these data have insufficient $\mathrm{S} / \mathrm{N}$ to test whether the stars in the offset envelope are a different stellar population from those in the symmetric halo.

\subsection{Tidal tails and streams around NGC 3311}

Comparison of tails in Hydra I with those around other galaxies - It is relevant to compare the luminosities of the streams in the Hydra I core with those observed in the halos of bright ellipticals in the Virgo cluster by Janowiecki et al. (2010). The HCC 026 stream identified here with a total luminosity slightly below $10^{9} L_{\odot, V}$ is significantly brighter than any of the Virgo streams, and the HCC 007 stream is several times more luminous still. In addition, the tidal streams seen around the Virgo cluster ellipticals have a much fainter peak surface brightness (brightest: $\mu_{V}=25.7 \mathrm{mag} \operatorname{arcsec}^{-2}$; average: $\mu_{V}=$ $27.6 \mathrm{mag} \mathrm{arcsec}^{-2}$ ) than those measured in the Hydra I streams, which are $\mu_{V}=24.8 \mathrm{mag} \mathrm{arcsec}^{-2}$ for the HCC 026 stream and $\mu_{V}=24.4 \mathrm{mag} \mathrm{arcsec}^{-2}$ for the HCC 007 stream.

Streams with as low a surface brightness as those observed in Virgo would not be detectable in our Hydra images, thus could easily be abundant in this region. The two small objects approximately near the middle of the polygon on the NE stream of HCC 007 in Fig. 11 may indeed be connected by a small stream within the HCC 007 tail, as suggested by the right panel of Fig. 9. This small stream, if confirmed, would have a comparable luminosity to the Virgo streams, albeit a higher surface brightness.

We can expand this comparison to the streams around bright local elliptical galaxies analyzed by Tal et al. (2009). The Hydra I streams are detected at radii larger than $\sim 23 \mathrm{kpc}$ from the center of NGC 3311, and the HCC 007 tail reaches at least $110 \mathrm{kpc}$ in total projected length (see the measurement in Sect. 4.1). These numbers for the Hydra I streams are consistent with the average radius of occurrence of the streams studied by Tal et al. (2009), and the linear extent of some of them. 
Unfortunately, Tal et al. (2009) do not give luminosities for their streams.

The streams are tidal tails - The streams emerging from HCC 026 and HCC 007 can be interpreted as the result of tidal disruption. In the case of HCC 007, the sheer size of the stream (the two tails together span $\sim 110 \mathrm{kpc}$ ) is difficult to explain by any other model. For HCC 026, the similar LOS velocity of the stream with the galaxy HCC 026 could still allow a model in which the stream stars are part of a shell that was stripped from a larger galaxy together with HCC 026. However, Coccato et al. (2011a) showed that the metallicity obtained with a single stellar population model in the stream region is lower than in the diffuse stellar halo of NGC 3311 away from the stream, and that this lower metallicity value is consistent with a composite population of stars from HCC 026 and stars from the stellar halo of NGC 3311 in approximately the proportion implied by the surface brightnesses of both components. This makes a strong case that the stars in the HCC 026 stream were indeed tidally stripped from HCC 026. It also shows that a fruitful way to investigate the origin of the diffuse light features in Hydra I and elsewhere is to measure stellar population parameters from deep spectroscopy.

Given their redshifted velocities, both the HCC 007 and HCC 026 streams would likely be located now on the distant side of the Hydra I cluster core, with their stars being on slightly different orbits than the galaxies they were once bound to.

Comparison with simulated tails - The overall morphology of the two streams in the Hydra I cluster core is similar to those of the tidal streams in the simulation investigated by Rudick et al. (2009) to study the formation of intracluster light. In particular, the tidal streams in their case G1 reproduce one characteristic property of the streams discovered here, especially for HCC 007 , that one side of the stream is significantly brighter than the other. In the simulation of Rudick et al. (2009), the $200 \mathrm{kpc}$ long tidal arms in G1 formed as a consequence of the interaction with the cluster $\mathrm{cD}$ of a disk galaxy, with a pericenter distance of $\sim 100 \mathrm{kpc}$. Most of the stars in the tidal streams in this system were unbound from the disk galaxy shortly after the pericentre passage, and once formed, the streams decayed in $\sim 1.5$ orbital times. The fact that tidal streams are visible around HCC 026 and HCC 007 thus indicates that both galaxies have recently passed the pericenters of their orbits.

\subsection{A group of galaxies in disruption in the Hydra I cluster core}

All the galaxies in disruption - As already reported by Ventimiglia et al. (2011), there are no galaxies in the Hydra I core at cluster-centric radii $(<100 \mathrm{kpc})$ with velocities around the cluster systemic velocity. However, several dwarf galaxies at high velocities $V_{\mathrm{LOS}} \geq 4500 \mathrm{~km} \mathrm{~s}^{-1}$ and the S0 galaxy HCC 007 are located in this region. In Table 3, we list the sky coordinates, apparent total $V$-band magnitudes, and LOS velocities for these galaxies from Misgeld et al. (2008). These galaxies are part of a well-defined cluster substructure in both velocity and spatial distribution, as already commented by Ventimiglia et al. (2011) and shown in Fig. 14. For two of these galaxies, HCC 026 and HCC 007, we have been able to detect tidal tails, showing that they are being disrupted by the tidal field near the cluster center. For the others, the tidal effects may not yet be strong enough to have led to detectable tidal tails, or their tails have dispersed below the detection limit. Independently of this, the observed tidal streams are the consequence of the recent infall of a cluster substructure containing the galaxies at $V_{\mathrm{LOS}}>4500 \mathrm{~km} \mathrm{~s}^{-1}$.

Where have the central galaxies gone? We now return to the observed absence of galaxies at the cluster systemic velocity in the central $100 \mathrm{kpc}$ around NGC 3311 (a similar result was found for the NGC 5044 group by Mendel et al. 2009). A plausible explanation is that these galaxies are no longer seen in the central region of the cluster because they were all disrupted in the past during close encounters with the central galaxy NGC 3311 and the dark matter cusp at the cluster center (Faltenbacher \& Mathews 2005). In this case, their former stars would now be part of the diffuse stellar component in the Hydra I core, including the halo around NGC 3311. It is interesting to ask whether most of the stars in the outer halo could have originated from the disruption of small galaxies in the way that seems to be currently ongoing, or whether the majority of the halo stars come through a different channel, namely the tidal disruption of the halos of massive elliptical galaxies prior to merging with the cluster $\mathrm{cD}$ (Murante et al. 2007; Puchwein et al. 2010). We highlight that the analysis of the stellar population properties of dwarf galaxies and the outer halo stars is important for answering this question (Coccato et al. 2010b, 2011a).

Phase-mixing and formation of ICL from the tidally dissolved stars - If we assumed that ultimately the interaction with NGC 3311 and the Hydra I cluster core completely disrupts the dwarf galaxies seen close to NGC 3311, all of their stars would phase-mix and be distributed in the central cluster potential. Converting the uni-directional velocity of their orbits of now $\sim 1200 \mathrm{~km} \mathrm{~s}^{-1}$ relative to NGC 3311 to $3 \sigma_{\text {eq }}^{2}$, the resulting $\sigma_{\mathrm{eq}} \simeq 700 \mathrm{~km} \mathrm{~s}^{-1}$. This would be somewhat increased by adding the contribution from the unknown transverse velocities. On the other hand, some of the kinetic energy of these stars would be converted to potential energy during the phase-mixing, lowering the final $\sigma_{\text {eq }}$. For comparison, the velocity dispersion of the NGC 3311 halo near the current position of HCC 026 is $\sigma_{\text {halo }} \simeq 400 \mathrm{~km} \mathrm{~s}^{-1}$. This suggests that the stars from the galaxies that are presently being tidally disrupted will end up at larger radii than their current position, and with somewhat larger dispersion than the observed $\sigma_{\text {halo }} \simeq 400 \mathrm{~km} \mathrm{~s}^{-1}$. That is, they will end up in the outermost halo and intracluster light around NGC 3311 and in the Hydra I cluster core.

\section{Summary and conclusions}

We have extended our previous investigation of the properties and origin of the diffuse light in the Hydra I cluster. Combining surface photometry with kinematic information from long-slit and planetary nebula (PN) data, we have found an off-centered, diffuse outer halo around the central galaxy NGC 3311, and showed that at least two galaxies are currently disrupted in the cluster core, adding their stars to the outer halo and intracluster light around NGC 3311. More specifically, our results are as follows.

Structural parameters have been derived for the two giant elliptical galaxies in the cluster core, NGC 3309 and NGC 3311, using $V$-band imaging data obtained at the ESO/MPI $2.2 \mathrm{~m}$ telescope and archival VLT/FORS1 $V$ band data. While the light distribution of NGC 3309 is reproduced by a single Sersic profile, that of NGC 3311 is characterized by several components. Outside the nuclear regions, which are affected by a dust lane and bright luminous knots, the bright regions within $30^{\prime \prime}$ follow an $R^{1 / 4}$ law. In the deep $V$ band data, these regions together with 
the symmetric part of the outer halo can be described by a Sersic law with $n \simeq 10$.

The residual image, obtained after subtracting the 2D model of the two bright galaxies, has revealed an additional extended envelope centered at $\sim 50^{\prime \prime}$ to the NE of NGC 3311. This offcentered envelope can be approximately described by an exponential profile and its luminosity is $L_{V, \mathrm{NE} \text {,env }}=1.2 \times 10^{10}( \pm 6.0 \times$ $\left.10^{8}\right) L_{\odot}$. This corresponds to $\sim 50 \%$ of the luminosity of the symmetric halo in the same region, and $\sim 15 \%$ of the luminosity of the entire symmetric halo in the radial range $25^{\prime \prime}-120^{\prime \prime}$.

Furthermore, the diffuse light in the Hydra I core harbors two tidal streams emerging from the dwarf galaxy HCC 026 and the S0 galaxy HCC 007 (see Fig. 11). The total luminosity in the NW part of the HCC 026 stream is $L_{V, \mathrm{NW}, \mathrm{HCC} 026}=$ $4.8 \times 10^{8}\left( \pm 8 \times 10^{7}\right) L_{\odot}$, with an average surface brightness $\mu_{V}=24.8 \pm 0.2 \mathrm{mag} \operatorname{arcsec}^{-2}$, which is about $15 \%$ of that in the extended envelope and symmetric halo at the stream position.

Our analysis of a deep spectrum in this region indicates that the NW stream has a LOS velocity similar to HCC 026 itself $\left(\sim 5000 \mathrm{~km} \mathrm{~s}^{-1}\right.$; see Sect. 5), and that it consists of stars with similar metallicities as the stars of HCC 026 (Coccato et al. 2011a). These results favor the interpretation that the HCC 026 stream has been tidally dissolved from HCC 026, over one where both the dwarf galaxy and the stream were dissolved from a larger galaxy. The luminosity of the combined NW and SE parts of the stream is several times the current luminosity of HCC 026 , $L_{V, \mathrm{HCC} 026}=1.5 \times 10^{8} L_{\odot}$, so that this dwarf galaxy has by now been mostly dissolved by the tidal field. Both HCC 026 and its tidal tails have a relative LOS velocity of $\sim 1200 \mathrm{~km} \mathrm{~s}^{-1}$ with respect to NGC $3311\left(V_{\mathrm{NGC} 3311} \simeq 3800 \mathrm{~km} \mathrm{~s}^{-1}\right)$.

The second tidal stream around the S0 galaxy HCC 007 extends over at least $\sim 110 \mathrm{kpc}$ on both sides of this galaxy. The tail is fairly thick and brighter on the NE side, where $\mu_{V}=24.4 \pm 0.5 \mathrm{mag} \operatorname{arcsec}^{-2}$. On the NW side, it is about one magnitude fainter and less certain. We measured a luminosity of $L_{V, \mathrm{NE} \text {, HCC } 007}=2.9 \times 10^{9}\left( \pm 5 \times 10^{8}\right) L_{\odot}$ in the brightest region of the NE tail. The NE tail appears to join the outer part of the offcentered halo east of NGC 3311; if so, its total luminosity could be significantly higher. For comparison, the total current luminosity of HCC 007 is $\sim 4.7 \times 10^{9} L_{\odot}$. This galaxy has therefore lost of order $50 \%$ of its stars.

Four PNs have been found in the NE HCC 007 tail, which allowed a preliminary measurement of its LOS velocity. The largest PN velocity $\left(5470 \mathrm{~km} \mathrm{~s}^{-1}\right)$ was found SE of NGC 3311 in a region dominated by tail stars. One PN is close to HCC 007 itself and has a velocity within $60 \mathrm{~km} \mathrm{~s}^{-1}$ of the galaxy's systemic velocity.

The morphologies of these streams are similar to those of tidal streams generated when galaxies are disrupted on highly radial orbits through the cluster center, shortly after the time of closest approach to the cluster cD (Rudick et al. 2009).

Superposed on the off-centered outer halo of NGC 3311, we have also found a number of PNs from the same subcomponent of the PN LOSVD centered at $5000 \mathrm{~km} \mathrm{~s}^{-1}$, as well as a group of dwarf galaxies with similar velocities. This suggests that there is a physical association between the off-centered halo, the two tidal streams, and an entire group of galaxies at about $5000 \mathrm{~km} \mathrm{~s}^{-1}$ systemic velocity, including HCC 026, several other dwarf galaxies, and HCC 007, which are currently falling through the core of the Hydra I cluster and have already been partially disrupted.

The fraction of the off-centered halo composed of stars dissolved from these galaxies is, however, poorly constrained by our measurements. The deep spectra show only a modest shift in mean LOS velocity in the NE halo, and the photometric, kinematic, and stellar population analyses of the HCC 026 tail suggests that the component at a $5000 \mathrm{~km} \mathrm{~s}^{-1}$ LOS velocity contributes only a fraction of the inferred off-centered halo at this location. Furthermore, a number of PNs with moderately to highly blue-shifted velocities are also seen superposed on the outer halo of NGC 3311 (Ventimiglia et al. 2011). To resolve this would require additional kinematical and stellar population analyses with deep spectroscopy at different positions in the halo.

This work provides a vivid example of how strong tidal forces cause morphological transformations of galaxies falling through the cores of galaxy clusters. It also shows that stars are currently being added to the diffuse light in the Hydra I cluster core. The stars in the streams around HCC 026 and HCC 007 must have been unbound from their parent galaxies during the ongoing close passage through the high-density center of the cluster, and now be on slightly different orbits from their once parent galaxies. Ultimately, these stars will phase-mix in the potential and add to both the diffuse outer envelope of NGC 3311 and the intracluster light in the cluster core.

The ongoing accretion of a group of galaxies will add about several $10^{9} L_{\odot, V}$ to the outer halo and intracluster light around NGC 3311, which is about $5 \%$ of the total light in the NGC 3311 symmetric halo. The tidal dissolution of small galaxies is thus an important channel for creating diffuse light in clusters. It is unlikely, however, that this is the main mechanism for the origin of the symmetric halo around NGC 3311. Both simulations (Murante et al. 2007; Puchwein et al. 2010) and studies of diffuse light in clusters such as Coma and the star pile cluster (Gerhard et al. 2007; Salinas et al. 2011) indicate that the disruption of and merger with of a giant galaxy on another similar galaxy is the more efficient route to creating large amounts of diffuse light. Deep spectroscopy to measure Lick indices and stellar abundances in different regions of the offset envelope and halo of NGC 3311 will be important in addressing this issue further.

Acknowledgements. The authors thank the referee for his/her report, the ESO VLT staff for their support during the observations, and Ken Freeman for helpful discussions during the course of this project. They also thank Ricardo Salinas and Tom Richtler for sending their NGC 3311 kinematic data in electronic form. E.I. acknowledges support from ESO during several visits while this work was completed. L.C. acknowledges funding from the European Community's Seventh Framework Programme (FP7/2007-2013/) under grant agreement No. 229517. This research has made use of the 2MASS data archive and the NASA/IPAC Extragalactic Database (NED), and of the ESO Science Archive Facility.

\section{References}

Arnaboldi, M., \& Gerhard, O. 2010, Highlights of Astronomy, 15, 97 Arnaboldi, M., Gerhard, O., Okamura, S., et al. 2007, PASJ, 59, 419 Buzzoni, A., Arnaboldi, M., \& Corradi, R. L. M. 2006, MNRAS, 368, 877 Cappellari, M., \& Emsellem, E. 2004, PASP, 116, 138

Ciardullo, R., Jacoby, G. H., Ford, H. C., \& Neill, J. D. 1989, ApJ, 339, 53 Coccato, L., Gerhard, O., Arnaboldi, M., et al. 2009, MNRAS, 394, 1249 Coccato, L., Arnaboldi, M., Gerhard, O., et al. 2010a, A\&A, 519, A95 Coccato, L., Gerhard, O., \& Arnaboldi, M. 2010b, MNRAS, 407, L26 Coccato, L., Gerhard, O., Arnaboldi, M., \& Ventimiglia, G. 2011a, A\&A, 533, A138

Coccato, L., Morelli, L., Corsini, E. M., et al. 2011b, MNRAS, 412, L113

De Lucia, G. 2007, in Cosmic Frontiers, eds. N. Metcalfe, \& T. Shanks, ASP Conf. Ser., 379, 257

Doherty, M., Arnaboldi, M., Das, P., et al. 2009, A\&A, 502, 771

Dolag, K., Murante, G., \& Borgani, S. 2010, MNRAS, 405, 1544

Faltenbacher, A., \& Mathews, W. G. 2005, MNRAS, 362, 498

Gerhard, O., Arnaboldi, M., Freeman, K. C., et al. 2005, ApJ, 621, L93

Gerhard, O., Arnaboldi, M., Freeman, K. C., et al. 2007, A\&A, 468, 815

Gonzalez, A. H., Zabludoff, A. I., \& Zaritsky, D. 2005, ApJ, 618, 195 
Hayakawa, A., Furusho, T., Yamasaki, N. Y., Ishida, M., \& Ohashi, T. 2004, PASJ, 56, 743

Hayakawa, A., Hoshino, A., Ishida, M., et al. 2006, PASJ, 58, 695 Jacoby, G. H. 1989, ApJ, 339, 39

Janowiecki, S., Mihos, J. C., Harding, P., et al. 2010, ApJ, 715, 972

Kelson, D. D., Zabludoff, A. I., Williams, K. A., et al. 2002, ApJ, 576, 720

Laine, S., van der Marel, R. P., Lauer, T. R., et al. 2003, AJ, 125, 478

McNeil, E. K., Arnaboldi, M., Freeman, K. C., et al. 2010, A\&A, 518, A44

Mendel, J. T., Proctor, R. N., Rasmussen, J., Brough, S., \& Forbes, D. A. 2009, MNRAS, 396, 2103

Mihos, J. C., Harding, P., Feldmeier, J., \& Morrison, H. 2005, ApJ, 631, L41

Misgeld, I., Mieske, S., \& Hilker, M. 2008, A\&A, 486, 697

Murante, G., Arnaboldi, M., Gerhard, O., et al. 2004, ApJ, 607, L83

Murante, G., Giovalli, M., Gerhard, O., et al. 2007, MNRAS, 377, 2

Murphy, J. D., Gebhardt, K., \& Adams, J. J. 2011, ApJ, 729, 129

Napolitano, N. R., Pannella, M., Arnaboldi, M., et al. 2003, ApJ, 594, 172

Poggianti, B. 2004, in Baryons in Dark Matter Halos, eds. R. Dettmar, U. Klein, \& P. Salucci
Puchwein, E., Springel, V., Sijacki, D., \& Dolag, K. 2010, MNRAS, 406, 936 Richtler, T., Salinas, R., Misgeld, I., et al. 2011, A\&A, 531, A119

Rudick, C. S., Mihos, J. C., Frey, L. H., \& McBride, C. K. 2009, ApJ, 699, 1518

Salinas, R., Richtler, T., West, M. J., et al. 2011, A\&A, 528, A61

Sanchez-Blazquez, P., Peletier, R. F., Jimenez-Vicente, J., et al. 2007, VizieR Online Data Catalog, 837, 10703

Schlegel, D. J., Finkbeiner, D. P., \& Davis, M. 1998, ApJ, 500, 525

Seigar, M. S., Graham, A. W., \& Jerjen, H. 2007, MNRAS, 378, 1575

Sommer-Larsen, J., Romeo, A. D., \& Portinari, L. 2005, MNRAS, 357, 478

Tal, T., van Dokkum, P. G., Nelan, J., \& Bezanson, R. 2009, AJ, 138, 1417

Thuan, T. X., \& Kormendy, J. 1977, PASP, 89, 466

Tonry, J., \& Davis, M. 1979, AJ, 84, 1511

Vasterberg, A. R., Lindblad, P. O., \& Jorsater, S. 1991, A\&A, 247, 335

Ventimiglia, G. 2011, Ph.D. Thesis, LMU München: Faculty of Physics

Ventimiglia, G., Arnaboldi, M., \& Gerhard, O. 2008, Astron. Nachr., 329, 1057

Ventimiglia, G., Gerhard, O., Arnaboldi, M., \& Coccato, L. 2010, A\&A, 520, L9

Ventimiglia, G., Arnaboldi, M., \& Gerhard, O. 2011, A\&A, 528, A24

Willman, B., Governato, F., Wadsley, J., \& Quinn, T. 2004, MNRAS, 355, 159 